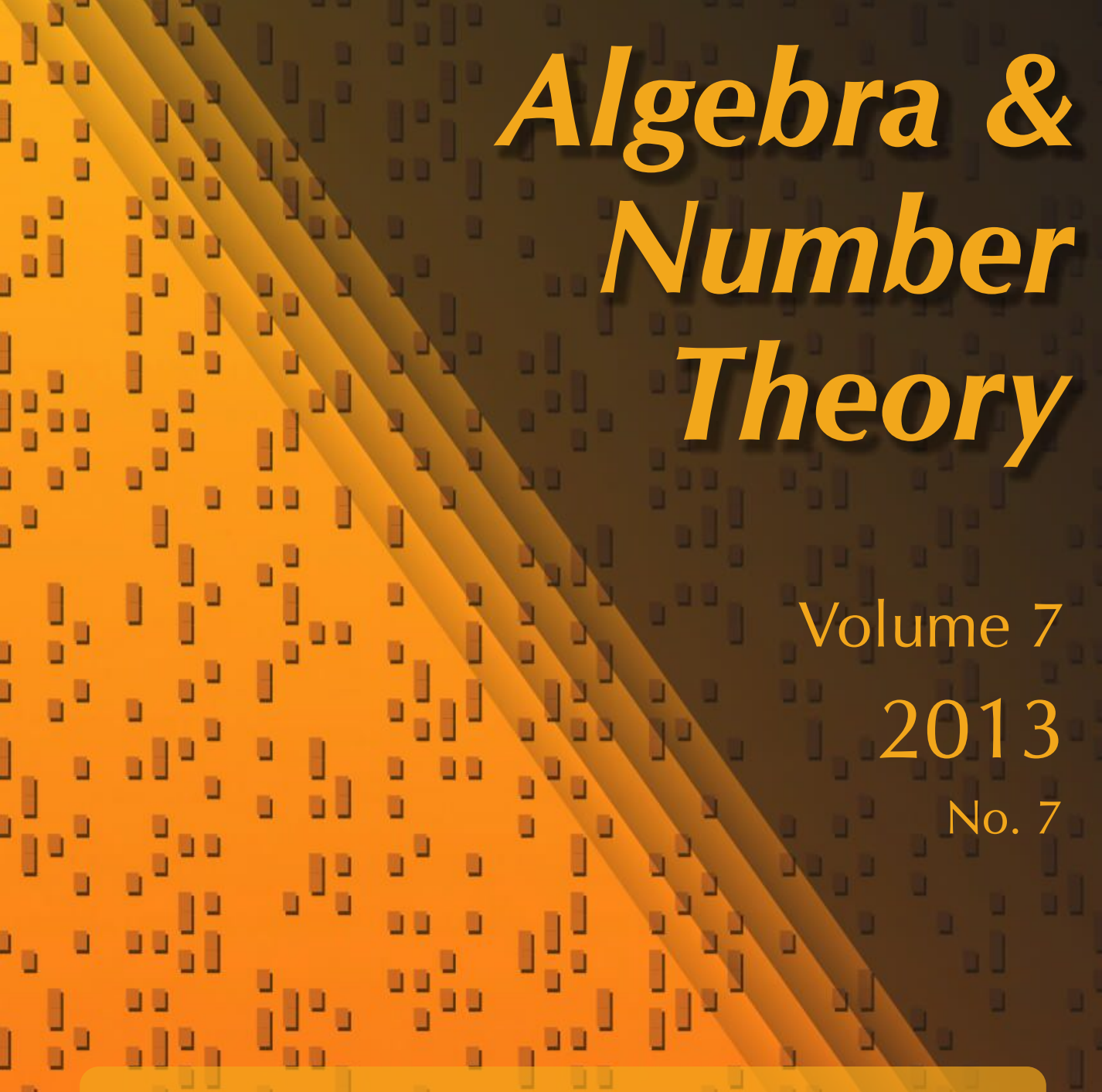




\title{
On abstract representations of the groups of rational points of algebraic groups and their deformations
}

\author{
Igor A. Rapinchuk
}

\begin{abstract}
In this paper, we continue our study, begun in an earlier paper, of abstract representations of elementary subgroups of Chevalley groups of rank $\geq 2$. First, we extend the methods to analyze representations of elementary groups over arbitrary associative rings and, as a consequence, prove the conjecture of Borel and Tits on abstract homomorphisms of the groups of rational points of algebraic groups for groups of the form $\mathbf{S L}_{n, D}$, where $D$ is a finite-dimensional central division algebra over a field of characteristic 0 . Second, we apply the previous results to study deformations of representations of elementary subgroups of universal Chevalley groups of rank $\geq 2$ over finitely generated commutative rings.
\end{abstract}

\section{Introduction and statement of the main results}

The goal of this paper is twofold. First, we extend the methods and results developed in our paper [Rapinchuk 2011] to analyze abstract representations of Chevalley groups over commutative rings to elementary groups over arbitrary associative rings. As a consequence of this analysis, we prove the conjecture of Borel and Tits [1973, 8.19] on abstract homomorphisms of the groups of rational points of algebraic groups for groups of the form $\mathbf{S L}_{n, D}$, where $D$ is a finite-dimensional central division algebra over a field of characteristic 0. Second, we apply the results of [Rapinchuk 2011] to study deformations of representations of the elementary subgroup $\Gamma=E(\Phi, R)$ of a universal Chevalley group associated to a root system $\Phi$ of rank $\geq 2$ over a finitely generated commutative ring $R$. This relies on the description, obtained in [Rapinchuk 2011], of representations with nonreductive image, which are at the heart of the Borel-Tits conjecture (recall that representations with reductive image were completely described in [Borel and Tits 1973]). We also use techniques of representation and character varieties (see [Lubotzky and Magid 1985]) in conjunction with the fact that such $\Gamma$ satisfies Kazhdan's property (T), which was recently established in [Ershov et al. 2011].

MSC2010: primary 20G15; secondary 14L15.

Keywords: abstract homomorphisms, algebraic groups, rigidity, character varieties. 
Before formulating of our first result, let us recall the statement of the Borel-Tits conjecture. For an algebraic $G$ defined over a field $k$, let $G^{+}$denote the subgroup of $G(k)$ generated by the $k$-points of split (smooth) connected unipotent $k$-subgroups.

Conjecture (BT). Let $G$ and $G^{\prime}$ be algebraic groups defined over infinite fields $k$ and $k^{\prime}$, respectively. If $\rho: G(k) \rightarrow G^{\prime}\left(k^{\prime}\right)$ is any abstract homomorphism such that $\rho\left(G^{+}\right)$is Zariski-dense in $G^{\prime}\left(k^{\prime}\right)$, then there exist a commutative finite-dimensional $k^{\prime}$-algebra $C$ and a ring homomorphism $f_{C}: k \rightarrow C$ such that $\rho=\sigma \circ r_{C / k^{\prime}} \circ F$, where $F: G(k) \rightarrow{ }_{C} G(C)$ is induced by $f_{C}\left({ }_{C} G\right.$ is the group obtained by change of scalars), $r_{C / k^{\prime}}:{ }_{C} G(C) \rightarrow R_{C / k^{\prime}}\left({ }_{C} G\right)\left(k^{\prime}\right)$ is the canonical isomorphism (here $R_{C / k^{\prime}}$ denotes the functor of restriction of scalars), and $\sigma$ is a rational $k^{\prime}$-morphism of $R_{C / k^{\prime}}\left({ }_{C} G\right)$ to $G^{\prime}$.

If an abstract homomorphism $\rho: G(k) \rightarrow G^{\prime}\left(k^{\prime}\right)$ admits a factorization as in (BT), we will say that $\rho$ has a standard description.

Remarks. (1) Another frequently used definition of $G^{+}$, which appears in the introduction of [Borel and Tits 1973], is that it is the subgroup of $G(k)$ generated by the $k$-points of the unipotent radicals of the parabolic $k$-subgroups of $G$. Recall that if $G$ is reductive, then the $k$-split smooth connected unipotent $k$-subgroups all lie in the unipotent radicals of minimal parabolic $k$-subgroups, so in this case, the two definitions coincide. However, they may differ for general smooth connected affine $k$-groups. Now, it follows from [Conrad et al. 2010, Proposition C.3.11, Theorem C.3.12] that in the case of a general smooth connected affine $k$-group $G$, one can also describe $G^{+}$as the subgroup of $G(k)$ generated by the $k$-points of the $k$-split unipotent radicals of the minimal pseudoparabolic $k$-subgroups.

(2) It was pointed out to us by B. Conrad and G. Prasad that, using techniques from the theory of pseudoreductive groups (developed in [Conrad et al. 2010, Chapter 9]), one can construct counterexamples to (BT) over all local and global function fields of characteristic 2 (or, more generally, over any field $k$ of characteristic 2 such that $\left.\left[k: k^{2}\right]=2\right)$. The groups that arise in these counterexamples are perfect and $k$-simple. So one should exclude fields of characteristic 2 (and possibly also characteristic 3 ) in the statement of (BT).

Our result concerning (BT) is as follows. Given a finite-dimensional central division algebra $D$ over a field $k$, we let $G=\mathbf{S L}_{n, D}$ denote the algebraic $k$-group such that $G(k)=\mathrm{SL}_{n}(D)$, the group of elements of $\mathrm{GL}_{n}(D)$ having reduced norm 1; recall that $G$ is an inner form of type $A_{l}$ (see [Knus et al. 1998; Platonov and Rapinchuk 1994] for details).

Theorem 1. Let $D$ be a finite-dimensional central division algebra over a field $k$ of characteristic 0 , and let $G=\mathbf{S L}_{n, D}$, where $n \geq 3$. Let $\rho: G(k) \rightarrow \operatorname{GL}_{m}(K)$ be a finite-dimensional linear representation of $G(k)$ over an algebraically closed 
field $K$ of characteristic 0 , and set $H=\overline{\rho(G(k))}$ (Zariski-closure). Then the abstract homomorphism $\rho: G(k) \rightarrow H(K)$ has a standard description.

In fact, we will see in Section 3 that a similar, but somewhat weaker, statement can be established for representations of elementary groups over arbitrary associative rings, not just division algebras (see Theorem 3.2 for a precise statement). It should be observed that while the overall structure of the proof of Theorem 1 resembles that of the Main Theorem of [Rapinchuk 2011], the analogs of the $K$-theoretic results of Stein [1973], which played a crucial role in [Rapinchuk 2011], were not available in the noncommutative setting. So part of our argument is dedicated to developing the required $K$-theoretic results, which is done in Section 2 using the computations of relative $K_{2}$ groups given by Bak and Rehmann [1982].

As we have already mentioned, results describing representations of a given group $\Gamma$ with nonreductive image can be used to analyze deformations of representations of $\Gamma$, which is the second major theme of this paper. Formally, over a field of characteristic 0 , deformations of (completely reducible) $n$-dimensional representations of a finitely generated group $\Gamma$ can be understood in terms of the corresponding character variety $X_{n}(\Gamma)$. For $\Gamma=E(\Phi, R)$, the elementary subgroup of $G(R)$, where $G$ is a universal Chevalley-Demazure group scheme corresponding to a reduced irreducible root system of rank $>1$ and $R$ is a finitely generated commutative ring, we use the results of [Rapinchuk 2011] to estimate the dimension of $X_{n}(\Gamma)$ as a function of $n$. (We note that it was recently shown in [Ershov et al. 2011] that such $\Gamma$ possesses Kazhdan's property $(\mathrm{T})$ and hence is finitely generated, so the representation variety $R_{n}(\Gamma)$ and the associated character variety $X_{n}(\Gamma)$ are defined. See Section 4 for a brief review of these notions and [Lubotzky and Magid 1985] for complete details.) To put our result into perspective, we recall that for $\Gamma=F_{d}$, the free group on $d>1$ generators, the dimension $\varkappa_{n}(\Gamma):=\operatorname{dim} X_{n}(\Gamma)$ is given by

$$
\varkappa_{n}(\Gamma)=(d-1) n^{2}+1,
$$

i.e., the growth of $\varkappa_{n}(\Gamma)$ is quadratic in $n$. It follows that the rate of growth cannot be more than quadratic for any finitely generated group (and it is indeed quadratic in some important situations such as $\Gamma=\pi_{g}$, the fundamental group of a compact orientable surface of genus $g>1$ [Rapinchuk et al. 1996]). At the other end of the spectrum are the groups $\Gamma$, called SS-rigid, for which $\varkappa_{n}(\Gamma)=0$ for all $n \geq 1$. For example, according to the superrigidity theorem of Margulis [1991, Chapter VII, Theorems 5.6, 5.25, and A], all irreducible higher-rank lattices are SS-rigid (see Section 5 regarding the superrigidity of groups like $E(\Phi, \mathcal{O})$, where $\mathbb{O}$ is a ring of algebraic integers). Now, in [Rapinchuk 2013], we show that if $\Gamma$ is not $S S$-rigid, then the rate of growth of $\varkappa_{n}(\Gamma)$ is at least linear. It follows that unless $\Gamma$ is $S S$-rigid, 
the growth rate of $\varkappa_{n}(\Gamma)$ is between linear and quadratic. Our result shows that for $\Gamma=E(\Phi, R)$ as above, this rate is the minimal possible, namely linear.

To formulate our result, we recall that a pair $(\Phi, R)$ consisting of a reduced irreducible root system of rank $>1$ and a commutative ring $R$ was called nice in [Rapinchuk 2011] if $2 \in R^{\times}$whenever $\Phi$ contains a subsystem of type $B_{2}$ and $2,3 \in R^{\times}$if $\Phi$ is of type $G_{2}$.

Theorem 2. Let $\Phi$ be a reduced irreducible root system of rank $\geq 2, R$ a finitely generated commutative ring such that $(\Phi, R)$ is a nice pair, and $G$ the universal Chevalley-Demazure group scheme of type $\Phi$. Let $\Gamma=E(\Phi, R)$ denote the elementary subgroup of $G(R)$, and consider the variety $X_{n}(\Gamma)$ of characters of $n$-dimensional representations of $\Gamma$ over an algebraically closed field $K$ of characteristic 0 . Then there exists a constant $c=c(R)$ (depending only on $R$ ) such that $\varkappa_{n}(\Gamma):=\operatorname{dim} X_{n}(\Gamma)$ satisfies

$$
\varkappa_{n}(\Gamma) \leq c \cdot n
$$

for all $n \geq 1$.

The proof is based on a suitable variation of the approach, going back to A. Weil, of bounding the dimension of the tangent space to $X_{n}(\Gamma)$ at a point $[\rho]$ corresponding to a representation $\rho: \Gamma \rightarrow \mathrm{GL}_{n}(K)$ by the dimension of the cohomology group $H^{1}\left(\Gamma, \operatorname{Ad}_{\mathrm{GL}_{n}} \circ \rho\right)$. Using the results of [Rapinchuk 2011], we describe the latter space in terms of certain spaces of derivations of $R$. This leads to the conclusion that the constant $c$ in Theorem 2 does not exceed the minimal number of generators $d$ of $R$ (i.e., the smallest integer such that there exists a surjection $\mathbb{Z}\left[X_{1}, \ldots, X_{d}\right] \rightarrow R$ ). In fact, if $R$ is the ring of integers or $S$-integers in a number field $L$, then $c=0$ (see Lemma 4.7), so we obtain that $\varkappa_{n}(\Gamma)=0$ for all $n$, i.e., $\Gamma$ is $S S$-rigid. We then show in Section 5 that the results of [Rapinchuk 2011] actually imply that $\Gamma=E(\Phi, R)$ is in fact superrigid in this case. The proof of Theorem 2 uses the validity of property $(\mathrm{T})$ for $\Gamma=E(\Phi, R)$. On the other hand, groups of this form account for most of the known examples of linear Kazhdan groups, so it is natural to ask if the conclusion of Theorem 2 can be extended to all discrete linear Kazhdan groups.

Conjecture. Let $\Gamma$ be a discrete linear group having Kazhdan's property $(T)$. Then there exists a constant $c=c(\Gamma)$ such that

$$
\varkappa_{n}(\Gamma) \leq c \cdot n
$$

for all $n \geq 1$.

The paper is organized as follows. In Section 2, we begin by summarizing some well known facts from $K$-theory and then use the results of [Bak and Rehmann 1982] to obtain a description of the group $K_{2}$ of certain associative rings similar to the one given by Stein in the commutative case. This is then used in the proof 
of Theorem 1, which is given in Section 3, along with similar results for arbitrary associative rings. Next, we begin Section 4 with a brief review of representation and character varieties and some related cohomological machinery, after which we turn to the proof of Theorem 2. Finally, in Section 5, we show how the techniques of [Rapinchuk 2011], along with some considerations involving derivations, can be used to establish various rigidity results for the elementary groups $E(\Phi, \mathcal{O})$, where $\mathcal{O}$ is a ring of algebraic integers.

Notations and conventions. Throughout the paper, $\Phi$ will denote a reduced irreducible root system of rank $\geq 2$. All of our rings are assumed to be associative and unital. As noted earlier, if $R$ is a commutative ring, we say that the pair $(\Phi, R)$ is nice if $2 \in R^{\times}$whenever $\Phi$ contains a subsystem of type $B_{2}$ and $2,3 \in R^{\times}$if $\Phi$ is of type $G_{2}$. Finally, given an algebraic group $H$, we let $H^{\circ}$ denote the connected component of the identity.

\section{2. $K$-theoretic preliminaries}

In this section, we develop the $K$-theoretic results that will be needed in the proof of Theorem 1. Even though the statements in this section are consequences of some well known results, to the best of our knowledge, they have never appeared explicitly in the literature in the form that we require. The main objective will be to use the computations of Bak and Rehmann [1982] to establish certain analogs in the noncommutative setting of Stein's [1973] description of the group $K_{2}$ of a semilocal commutative ring (see Propositions 2.3 and 2.4 below).

We begin by recalling some standard definitions. Let $R$ be an associative unital ring. For $1 \leq i, j \leq n, i \neq j$, and $r \in R$, let $e_{i j}(r) \in \mathrm{GL}_{n}(R)$ be the elementary matrix with $r$ in the $(i, j)$-th place, and let $E_{n}(R)$ denote the subgroup of $\mathrm{GL}_{n}(R)$, called the elementary group, generated by all the $e_{i j}(r)$. If $n \geq 3$, it is well known that the elementary matrices in $\mathrm{GL}_{n}(R)$ satisfy the following relations:

(R1) $e_{i j}(r) e_{i j}(s)=e_{i j}(r+s)$.

(R2) $\left[e_{i j}(r), e_{k l}(s)\right]=1$ if $i \neq l$ and $j \neq k$.

(R3) $\left[e_{i j}(r), e_{j l}(s)\right]=e_{i l}(r s)$ if $i \neq l$.

The Steinberg group over $R$, denoted $\mathrm{St}_{n}(R)$, is defined to be the group generated by all symbols $x_{i j}(r)$ with $1 \leq i, j \leq n, i \neq j$, and $r \in R$ subject to the natural analogs of the relations (R1)-(R3) written in terms of the $x_{i j}(r)$. From the definition, it is clear that there exists a canonical surjective group homomorphism

$$
\pi_{R}: \mathrm{St}_{n}(R) \rightarrow E_{n}(R), \quad x_{i j}(r) \mapsto e_{i j}(r),
$$

and we set

$$
K_{2}(n, R)=\operatorname{ker}\left(\mathrm{St}_{n}(R) \stackrel{\pi_{R}}{\longrightarrow} E_{n}(R)\right)
$$


It is easy to see that there exist natural homomorphisms $\operatorname{St}_{n}(R) \rightarrow \mathrm{St}_{n+1}(R)$ and $E_{n}(R) \hookrightarrow E_{n+1}(R)$, which induce homomorphisms $K_{2}(n, R) \rightarrow K_{2}(n+1, R)$ [Hahn and O'Meara 1989, §1.4]. Also notice that the pair $\left(\operatorname{St}_{n}(R), \pi_{R}\right)$ is functorial in the following sense: given a homomorphism of rings $f: R \rightarrow S$, there is a commutative diagram of group homomorphisms

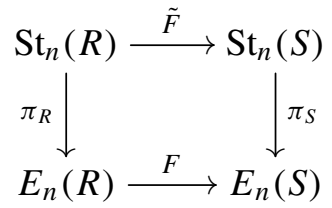

where $F$ and $\tilde{F}$ are the homomorphisms induced by $f$ defined on generators by

$$
F: e_{i j}(t) \mapsto e_{i j}(f(t)) \quad \text { and } \quad \tilde{F}: x_{i j}(t) \mapsto x_{i j}(f(t)) .
$$

It follows from the commutativity of the above diagram that $\tilde{F}$ induces a homomorphism $K_{2}(n, R) \rightarrow K_{2}(n, S)$. In the following proposition, we derive some general properties of $K_{2}(n, R)$ that will be needed later in this section:

Proposition 2.1. (a) Suppose $R$ is an associative unital ring such that $R / J(R)$ is artinian, where $J(R)$ is the Jacobson radical of $R$. Then the natural map $K_{2}(3, R) \rightarrow K_{2}(4, R)$ is an isomorphism. If, moreover, $R$ is finitely generated as a module over its center, then $K_{2}(n, R)$ is a central subgroup of $\operatorname{St}_{n}(R)$ for $n \geq 3$.

(b) Suppose $C$ is a commutative finite dimensional algebra over a field $K$, and let $A=M_{m}(C)$ be the ring of $m \times m$ matrices over $C$. For $a \in C$ and $1 \leq k, l \leq m$, let $\tilde{y}_{k l}(a) \in A$ be the matrix with a as the $(k, l)$ entry and 0 for all other entries. Then for $n \geq 3$, the maps

$$
\begin{aligned}
& \tilde{\psi}\left(x_{i j}^{A}\left(\tilde{y}_{k l}(a)\right)\right)=x_{(i-1) m+k,(j-1) m+l}^{C}(a), \\
& \psi\left(e_{i j}^{A}\left(\tilde{y}_{k l}(a)\right)\right)=e_{(i-1) m+k,(j-1) m+l}^{C}(a),
\end{aligned}
$$

where the $x_{i j}^{A}(a)$ and $e_{i j}^{A}(a)$ are the generators of $\operatorname{St}_{n}(A)$ and $E_{n}(A)$ and the $x_{i j}^{C}(c)$ and $e_{i j}^{C}(c)$ are the generators of $\operatorname{St}_{n m}(C)$ and $E_{n m}(C)$, respectively, define isomorphisms $\tilde{\psi}: \mathrm{St}_{n}(A) \rightarrow \mathrm{St}_{n m}(C)$ and $\psi: E_{n}(A) \rightarrow E_{n m}(C)$ such that the following diagram commutes:

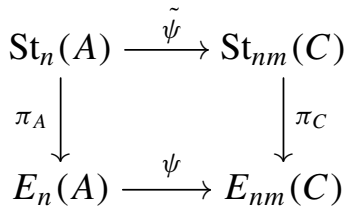

In particular, $K_{2}\left(n, M_{m}(C)\right) \simeq K_{2}(n m, C)$. 
Proof. (a) By Theorem 7 of [van der Kallen 1976], the fact that $R / \operatorname{Rad}(R)$ is artinian implies that it has property $S R_{2}^{*}$, and then Theorem 6 of the same work yields the required isomorphism. Now, if $R$ is finitely generated as a module over its center, then according to [Hahn and O'Meara 1989, Theorem 1.4.15], $\pi_{R}: \operatorname{St}_{n}(R) \rightarrow E_{n}(R)$ is a central extension for $n \geq 4$ (in fact, a universal central extension for $n \geq 5$ ). So in view of the canonical isomorphism $K_{2}(3, R) \simeq K_{2}(4, R)$, we obtain that $K_{2}(n, R)$ is a central subgroup of $\operatorname{St}_{n}(R)$ for $n \geq 3$, as claimed.

(b) First notice that the natural group isomorphism $\mathrm{GL}_{n}(A) \stackrel{\sim}{\rightarrow} \mathrm{GL}_{n m}(C)$ restricts to a group homomorphism $\psi: E_{n}(A) \rightarrow E_{n m}(C)$. By direct computation with commutator relations, one sees that $\psi$ is surjective for $n \geq 3$ and hence an isomorphism. Moreover, on generators it is given by the second formula in the statement. Now, since $A$ is generated additively by the $\tilde{y}_{k l}(a)$, with $1 \leq k, l \leq m$, it follows that the $\tilde{x}_{i j}^{A}\left(\tilde{y}_{k l}(a)\right)$ generate $\mathrm{St}_{n}(A)$, so it suffices to define $\tilde{\psi}$ on these elements and check the defining relations. This is done by direct computation using the definition of $\tilde{\psi}$ given above.

Next, since without loss of generality $m \geq 2$, we have $n m \geq 6$, so as noted in the proof of (a), $\pi_{C}: \mathrm{St}_{n m}(C) \rightarrow E_{n m}(C)$ is a universal central extension and $\pi_{A}: \operatorname{St}_{n}(A) \rightarrow E_{n}(A)$ is a central extension. Hence, there exists a unique group homomorphism $\tilde{\varphi}: S t_{n m}(C) \rightarrow \mathrm{St}_{n}(A)$ making the diagram

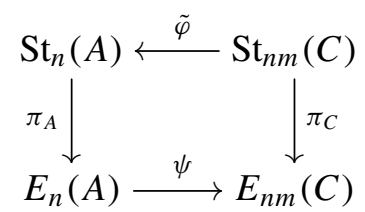

commute, and by universality, we conclude that $\tilde{\psi} \circ \tilde{\varphi}=\mathrm{id}_{\mathrm{St}_{n m}(C)}$. On the other hand, by the commutativity of the diagrams (1) and (2), we have that for any $x \in \operatorname{St}_{n}(A)$,

$$
\left(\psi \circ \pi_{A} \circ \tilde{\varphi} \circ \tilde{\psi}\right)(x)=\left(\pi_{C} \circ \tilde{\psi}\right)(x)=\left(\psi \circ \pi_{A}\right)(x) .
$$

Since $\psi$ is an isomorphism, we conclude that $(\tilde{\varphi} \circ \tilde{\psi})(x)=x z_{x}$, where $z_{x} \in K_{2}(n, A)$. The centrality of $K_{2}(n, A)$ then implies that the map $x \mapsto z_{x}$ is a homomorphism $\operatorname{St}_{n}(A) \rightarrow K_{2}(n, A)$, which must be trivial as $\operatorname{St}_{n}(A)$ is a perfect group and $K_{2}(n, A)$ is commutative. Thus, $\tilde{\varphi} \circ \tilde{\psi}=\mathrm{id}_{\mathrm{St}_{n}(A)}$, as required. It then follows that $K_{2}(n, A) \simeq K_{2}(n m, C)$.

Next, let us summarize the results of [Bak and Rehmann 1982] dealing with relative $K_{2}$ groups of associative rings (see Theorem 2.2 below). From now on, we will always assume that $n \geq 3$. First, we need to introduce some additional notation. As above, let $R$ be an associative unital ring. Given $u \in R^{\times}$, we define, for $i \neq j$, 
the following standard elements of $\operatorname{St}_{n}(R)$ :

$$
w_{i j}(u)=x_{i j}(u) x_{j i}\left(-u^{-1}\right) x_{i j}(u) \quad \text { and } \quad h_{i j}(u)=w_{i j}(u) w_{i j}(-1) .
$$

Notice that the image $\pi_{R}\left(h_{i j}(u)\right)$ in $E_{n}(R)$ is the diagonal matrix with $u$ as the $i$-th diagonal entry, $u^{-1}$ as the $j$-th diagonal entry, and 1 s everywhere else on the diagonal. We will also need the following noncommutative version of the usual Steinberg symbols: for $u, v \in R^{\times}$, let

$$
c(u, v)=h_{12}(u) h_{12}(v) h_{12}(v u)^{-1} .
$$

One easily sees that $\pi_{R}(c(u, v))$ is the diagonal matrix with $u v u^{-1} v^{-1}$ as its first diagonal entry and $1 \mathrm{~s}$ everywhere else on the diagonal. Let $U_{n}(R)$ be the subgroup of $\mathrm{St}_{n}(R)$ generated by all the $c(u, v)$ with $u, v \in R^{\times}$.

As in the commutative case, one can also consider relative versions of these constructions. Let $\mathfrak{a}$ be a two-sided ideal of $R$ and

$$
\mathrm{GL}_{n}(R, \mathfrak{a})=\operatorname{ker}\left(\mathrm{GL}_{n}(R) \rightarrow \mathrm{GL}_{n}(R / \mathfrak{a})\right)
$$

be the congruence subgroup of level $\mathfrak{a}$. Define $E_{n}(R, \mathfrak{a})$ to be the normal subgroup of $E_{n}(R)$ generated by all elementary matrices $e_{i j}(a)$ with $a \in \mathfrak{a}$. Now letting

$$
\operatorname{St}_{n}(R, \mathfrak{a})=\operatorname{ker}\left(\operatorname{St}_{n}(R) \rightarrow \operatorname{St}_{n}(R / \mathfrak{a})\right),
$$

we have a natural homomorphism $\operatorname{St}_{n}(R, \mathfrak{a}) \rightarrow E_{n}(R, \mathfrak{a})$, and we set

$$
K_{2}(n, R, \mathfrak{a})=\operatorname{ker}\left(\operatorname{St}_{n}(R, \mathfrak{a}) \rightarrow E_{n}(R, \mathfrak{a})\right) .
$$

Finally, let

$$
U_{n}(R, \mathfrak{a}):=\left\langle c(u, 1+a) \mid u \in R^{\times}, 1+a \in(1+\mathfrak{a}) \cap R^{\times}\right\rangle
$$

(notice this is contained in $\operatorname{St}_{n}(R, \mathfrak{a})$ ). We should point out that even though for a noncommutative ring, the groups $U_{n}(R)$ and $U_{n}(R, \mathfrak{a})$ may not lie in $K_{2}(n, R)$, it is well known that any element of $K_{2}(n, R) \cap U_{n}(R)$ is automatically contained in the center of $\operatorname{St}_{n}(R)$ [Milnor 1971, Corollary 9.3]. This will be needed in Proposition 2.3 below.

Theorem 2.2 [Bak and Rehmann 1982, Theorem 2.9, Corollary 2.11]. Let $R$ be an associative unital ring. Suppose that $\mathfrak{a}$ is a two-sided ideal contained in the Jacobson radical $J(R)$ of $R$ and that $R$ is additively generated by $R^{\times}$. Assume $n \geq 3$. Then the following are true:

(1) $K_{2}(n, R, \mathfrak{a}) \subset U_{n}(R, \mathfrak{a})$, and the canonical sequence below is exact:

$$
1 \rightarrow U_{n}(R, \mathfrak{a}) \rightarrow U_{n}(R) \rightarrow U_{n}(R / \mathfrak{a}) \rightarrow 1 .
$$


(2) If, moreover, $K_{2}(n, R / \mathfrak{a}) \subset U_{n}(R / \mathfrak{a})$, then $K_{2}(n, R) \subset U_{n}(R)$ and the natural sequence

$$
1 \rightarrow K_{2}(n, R, \mathfrak{a}) \rightarrow K_{2}(n, R) \rightarrow K_{2}(n, R / \mathfrak{a}) \rightarrow 1
$$

is exact.

The theorem yields the following:

Proposition 2.3. Suppose that $R$ is either a finite-dimensional algebra over an algebraically closed field $K$ or a finite ring with $2 \in R^{\times}$. Then $K_{2}(n, R) \subset U_{n}(R)$, and consequently, $K_{2}(n, R)$ is a central subgroup of $\operatorname{St}_{n}(R)$.

Proof. Let $J=J(R)$ be the Jacobson radical of $R$. To apply Theorem 2.2, we need to verify that in both cases, $R$ is additively generated by its units and that $K_{2}(n, R / J) \subset U_{n}(R / J)$.

If $R$ is a finite-dimensional algebra over $K$, then we can view $R$ as a connected algebraic ring over $K$, and it follows from [Rapinchuk 2011, Corollary 2.5] that $R$ is generated by $R^{\times} \cdot{ }^{1}$ Now suppose that $R$ is a finite ring. Since $R$ is obviously artinian, $R / J$ is semisimple [Lam 2001, Theorem 4.14], so by the Artin-Wedderburn theorem [Lam 2001, Theorem 3.5] and the fact that finite division rings are commutative [Lam 2001, Theorem 13.1], we have

$$
R / J \simeq M_{n_{1}}\left(F_{1}\right) \oplus \cdots \oplus M_{n_{r}}\left(F_{r}\right)
$$

where $F_{1}, \ldots, F_{r}$ are finite fields with $F_{i} \neq \mathbb{F}_{2}$, the field of two elements, for all $i$ as $2 \in R^{\times}$. It follows that $R / J$ is additively generated by its units. On the other hand, the canonical map $R \rightarrow R / J$ induces a surjective homomorphism $R^{\times} \rightarrow(R / J)^{\times}$, which, combined with the fact that $J$ lies in the linear span of $R^{\times}$[Lam 2001, Lemma 4.3], yields that $R$ is additively generated by $R^{\times}$.

Next, let us show that $K_{2}(n, R / J) \subset U_{n}(R / J)$ in both cases. If $R$ is a finitedimensional $K$-algebra, then as above $R / J$ is semisimple. So since there are no nontrivial division algebras over algebraically closed fields, the Artin-Wedderburn theorem implies that

$$
R / J \simeq M_{n_{1}}(K) \oplus \cdots \oplus M_{n_{s}}(K)
$$

Thus, in both cases, $R / J$ is a direct sum of matrix algebras over fields. Since $K_{2}$ commutes with finite direct sums, we may assume without loss of generality that $A:=R / J \simeq M_{m}(F)$ with $F$ a field. By Proposition 2.1, we have isomorphisms $\tilde{\psi}: \mathrm{St}_{n}(A) \rightarrow \mathrm{St}_{n m}(F)$ and $\psi: E_{n}(A) \rightarrow E_{n m}(F)$ that induce an isomorphism

${ }^{1}$ All the background on algebraic rings needed in this paper can be found in [Rapinchuk 2011, §2]. M. Kassabov has also informed us that the notion of an algebraic ring actually goes back to [Greenberg 1964], where one can find proofs of some basic properties. 
$K_{2}(n, A) \simeq K_{2}(n m, F)$. Now let $u \in F^{\times}$and $t_{u}=\operatorname{diag}(u, 1, \ldots, 1) \in M_{m}(F)$. By direct computation, one checks that

$$
\tilde{\psi}\left(h_{12}^{A}\left(t_{u}\right)\right)=h_{1, m+1}^{F}(u),
$$

and therefore, for $u, v \in F^{\times}$, we have

$$
\tilde{\psi}\left(c\left(t_{u}, t_{v}\right)\right)=c_{1, m+1}(u, v),
$$

where $c_{1, m+1}(u, v)=h_{1, m+1}^{F}(u) h_{1, m+1}^{F}(v) h_{1, m+1}^{F}(v u)^{-1}$. On the other hand, by Matsumoto's theorem, the group $K_{2}(n m, F)$ is generated by the Steinberg symbols $c_{1, m+1}(u, v)$ [Steinberg 1968]; consequently, we see $K_{2}(n, R / J) \subset U_{n}(R / J)$, as claimed. Hence, $K_{2}(n, R) \subset U_{n}(R)$ by Theorem 2.2. As noted above, it now follows from [Milnor 1971, Corollary 9.3] that $K_{2}(n, R)$ lies in the center of $\operatorname{St}_{n}(R)$.

An important ingredient in the proof of Theorem 1 will be the following:

Proposition 2.4. Let $k$ and $K$ be fields of characteristic 0 with $K$ algebraically closed. Suppose that $D$ is a finite-dimensional central division algebra over $k, A$ a finite-dimensional algebra over $K$, and $f: D \rightarrow A$ a ring homomorphism with Zariski-dense image. Then for $n \geq 3, K_{2}(n, A)$ coincides with the subgroup

$$
U_{n}^{\prime}(A)=\left\langle c(u, v) \mid u, v \in \overline{f\left(L^{\times}\right)}\right\rangle,
$$

where $L$ is an arbitrary maximal subfield of $D$.

We begin with the following:

Lemma 2.5. Let $A, D$, and $f$ be as above, and set $C=\overline{f(k)}$ (Zariski closure). Then

$$
A \simeq D \otimes_{k} C \simeq M_{s}(C)
$$

as $K$-algebras, where $s^{2}=\operatorname{dim}_{k} D$. Moreover, if $L$ is any maximal subfield of $D$, then the second isomorphism can be chosen so that $L \otimes_{k} C \simeq D_{s}(C)$, where $D_{s}(C) \subset M_{s}(C)$ is the subring of diagonal matrices.

Proof. We start with the proof of the first isomorphism in (3). To begin, we note that since $k$ and $K$ are both fields of characteristic $0, C$ is a finite-dimensional algebra over $K$ by [Rapinchuk 2011, Lemma 2.13, Proposition 2.14]. Moreover, by [Greenberg 1964, Proposition 5.1], the natural inclusion $C \hookrightarrow A$ is a homomorphism of $K$-algebras (this also follows from the proof of [Rapinchuk 2011, Proposition 2.14]). Now consider the map

$$
\theta: D \otimes_{k} C \rightarrow A, \quad(x, c) \mapsto f(x) c .
$$

We claim that $\theta$ is an isomorphism. From the above remark, it is clear that $\theta$ is a homomorphism of $K$-algebras (where $D \otimes_{k} C$ is endowed with the natural $K$-algebra structure coming from $C$ ). For surjectivity, first note that since $\operatorname{im} \theta$ 
contains $f(D)$, it is Zariski-dense in $A$. On the other hand, let $x_{1}, \ldots, x_{s^{2}}$ be a basis of $D$ over $k$. Then

$$
\operatorname{im} \theta=f\left(x_{1}\right) C+\cdots+f\left(x_{s^{2}}\right) C
$$

and therefore is closed. Hence, $\theta$ is surjective. To prove injectivity, notice that since $D$ is a central simple algebra, $\operatorname{ker} \theta=D \otimes_{k} \mathfrak{c}$ for some ideal $\mathfrak{c} \subset C$ [Farb and Dennis 1993, Theorem 3.5]. On the other hand, since by construction the restriction $\left.\theta\right|_{\mathfrak{c}}$ is an embedding, we have $\mathfrak{c}=0$, and $\theta$ is injective.

Now let us consider the second isomorphism. First, since $C$ is a commutative artinian algebraic ring, by [Rapinchuk 2011, Proposition 2.20], we can write

$$
C=C_{1} \times \cdots \times C_{r},
$$

where each $C_{i}$ is a local commutative algebraic ring. Moreover, since tensor products commute with finite products and $M_{s}\left(C_{1} \times \cdots \times C_{r}\right)=M_{s}\left(C_{1}\right) \times \cdots \times M_{s}\left(C_{r}\right)$, it suffices to establish the isomorphism when $C$ is a local algebraic ring. So suppose that is the case, and let $J(C)$ be the Jacobson radical of $C$. Then it follows from [Rapinchuk 2011, Corollary 2.6, Proposition 2.19] that $C / J(C) \simeq K$, so composing $f$ with the canonical map $C \rightarrow C / J(C)$, we obtain an embedding $k \hookrightarrow K$. Consequently, as $K$ is algebraically closed, the division algebra $D$ splits over $K$, i.e., there exists an isomorphism

$$
\tau: D \otimes_{k} K \stackrel{\sim}{\rightarrow} M_{S}(K) .
$$

Notice also if $L$ is a maximal subfield of $D$, we can choose $\tau$ so that $L \otimes_{k} K \simeq D_{s}(K)$. Indeed, since $L$ is separable over $k$ (as char $k=0$ ) and $[L: k]=s$, we can write $L=k[X] /(f)$, where $f$ is a separable polynomial of degree $s$. Then by the Chinese remainder theorem, $L \otimes_{k} K \simeq K^{s}$. But any subalgebra of $M_{s}(K)$ that is isomorphic to $K^{s}$ is conjugate to $D_{s}(K)$ [Gille and Szamuely 2006, Lemma 2.2.9], so it follows that $\tau$ can be composed with an inner automorphism of $M_{S}(K)$ to have the required form.

Now consider the natural (surjective) map

$$
D \otimes_{k} C \rightarrow D \otimes_{k}(C / J(C))=D \otimes_{k} K .
$$

Since $D$ is a central simple algebra, the same argument as above shows that the kernel of this map is contained in the Jacobson radical $J\left(D \otimes_{k} C\right)$, and the fact that $D \otimes_{k} K \simeq M_{s}(K)$ is semisimple implies that it actually coincides with $J\left(D \otimes_{k} C\right)$. So by the Wedderburn-Malcev theorem [Pierce 1982, Corollary 11.6], there exists a section

$$
\alpha: M_{s}(K) \simeq D \otimes_{k} K \hookrightarrow D \otimes_{k} C
$$


We claim that the following map gives the required isomorphism:

$$
\beta: M_{s}(K) \otimes_{K} C \rightarrow D \otimes_{k} C, \quad m \otimes c \mapsto \alpha(m) \cdot(1 \otimes c) .
$$

Indeed, injectivity is proved by the same argument as above, and surjectivity follows by dimension count. Thus, $M_{s}(C) \simeq M_{s}(K) \otimes_{K} C \simeq D \otimes_{k} C$, and it follows immediately from the above remarks that $D_{s}(C) \simeq L \otimes_{k} C$.

Proof of Proposition 2.4. By Lemma 2.5, we have $L \otimes_{k} C \simeq D_{s}(C)$. Moreover, $L \otimes_{k} C \simeq \overline{f(L)}$. Indeed, since $k \subset L$, we have

$$
f(L) \subset \theta\left(L \otimes_{k} C\right) \subset \overline{f(L)} .
$$

On the other hand, the same argument as in the proof of Lemma 2.5 shows that $\theta\left(L \otimes_{k} C\right)$ is closed.

Next, since $A \simeq M_{S}(C)$ and $C$ is a finite-dimensional $K$-algebra, there exists by Proposition 2.1 an isomorphism $\tilde{\psi}: \mathrm{St}_{n}(A) \rightarrow \mathrm{St}_{n s}(C)$ that induces an isomorphism $K_{2}(n, A) \simeq K_{2}(n s, C)$. Now, $C$ is a semilocal commutative ring that is additively generated by its units, so $K_{2}(n s, C)$ coincides with the subgroup $U_{n s}(C)$ of $\mathrm{St}_{n s}(C)$ generated by the Steinberg symbols $c_{1, s+1}(u, v)$ taken with respect to the root $\alpha_{1, s+1}$ (i.e., $\left.c_{1, s+1}(u, v)=h_{1, s+1}(u) h_{1, s+1}(v) h_{1, s+1}(v u)^{-1}\right)$ by [Stein 1973, Theorem 2.13]. As we noted in the proof of Proposition 2.3, we have

$$
\tilde{\psi}\left(c\left(t_{u}, t_{v}\right)\right)=c_{1, s+1}(u, v),
$$

where for $u \in C^{\times}$, we set $t_{u}=\operatorname{diag}(u, 1, \ldots, 1) \in M_{s}(C)$. Thus, $K_{2}(n, A)$ is contained in the group generated by the symbols $c\left(t_{u}, t_{v}\right)$. On the other hand, since all of the $t_{u}$ are diagonal matrices, they lie in the image of $L \otimes_{k} C$; hence, $K_{2}(n, A) \subset U_{n}^{\prime}(A)$. Since clearly $U_{n}^{\prime}(A) \subset K_{2}(n, A)$, this concludes the proof.

\section{Abstract homomorphisms over noncommutative rings}

The main goal of this section is to give the proof of Theorem 1. Before beginning the argument, we would like to give an alternative statement of Theorem 1, which can be generalized (in a somewhat weaker form) to (essentially) arbitrary associative rings. First, we need to observe that if $B$ is a finite-dimensional algebra over an algebraically closed field $K$, then the elementary group $E_{n}(B)$ has the structure of a connected algebraic $K$-group. Indeed, using the regular representation of $B$ over $K$, it is easy to see that $\mathrm{GL}_{n}(B)$ is a Zariski-open subset of $M_{n}(B)$ and hence an algebraic group over $K$. Now let us view $B$ as a connected algebraic ring over $K$, and for $i, j \in\{1, \ldots, n\}, i \neq j$, consider the regular maps

$$
\varphi_{i j}: B \rightarrow \mathrm{GL}_{n}(B), \quad b \mapsto e_{i j}(b) .
$$


Set $W_{i j}=\operatorname{im} \varphi_{i j}$. Then each $W_{i j}$ contains the identity matrix $I_{n} \in \operatorname{GL}_{n}(B)$, and by definition, $E_{n}(B)$ is generated by the $W_{i j}$. So $E_{n}(B)$ is a connected algebraic group by [Borel 1991, Proposition 2.2].

Theorem 3.1. Suppose $k$ and $K$ are fields of characteristic 0 with $K$ algebraically closed, $D$ is a finite-dimensional central division algebra over $k$, and $n$ is an integer $\geq 3$. Let $\rho: E_{n}(D) \rightarrow \mathrm{GL}_{m}(K)$ be a finite-dimensional linear representation, and set $H=\overline{\rho\left(E_{n}(D)\right)}$ (Zariski closure). Then there exist a finite-dimensional associative $K$-algebra $\mathscr{B}$, a ring homomorphism $f: D \rightarrow \mathscr{B}$ with Zariski-dense image, and a morphism $\sigma: E_{n}(\mathscr{B}) \rightarrow H$ of algebraic $K$-groups such that

$$
\rho=\sigma \circ F,
$$

where $F: E_{n}(D) \rightarrow E_{n}(\mathscr{B})$ is the group homomorphism induced by $f$.

We also have the following result for general associative rings:

Theorem 3.2. Suppose $R$ is an associative ring with $2 \in R^{\times}, K$ is an algebraically closed field of characteristic 0 , and $n$ is an integer $\geq 3$. Let $\rho: E_{n}(R) \rightarrow \operatorname{GL}_{m}(K)$ be a finite-dimensional linear representation, set $H=\overline{\rho\left(E_{n}(R)\right)}$, and let $H^{\circ}$ denote the connected component of $H$. If the unipotent radical of $H^{\circ}$ is commutative, there exist a finite-dimensional associative $K$-algebra $\mathscr{B}$, a ring homomorphism $f: R \rightarrow \mathscr{B}$ with Zariski-dense image, and a morphism $\sigma: E_{n}(\mathscr{B}) \rightarrow H$ of algebraic $K$-groups such that for a suitable finite-index subgroup $\Delta \subset E_{n}(R)$, we have

$$
\left.\rho\right|_{\Delta}=\left.(\sigma \circ F)\right|_{\Delta},
$$

where $F: E_{n}(R) \rightarrow E_{n}(\mathscr{B})$ is the group homomorphism induced by $f$.

As we indicated in the introduction, the proofs of Theorems 3.1 and 3.2 are based on a natural extension of the approach developed in our earlier paper [Rapinchuk 2011]. More precisely, we will first associate to $\rho$ an algebraic ring $A$, then show that $\rho$ can be lifted to a representation $\tilde{\tau}: \mathrm{St}_{n}(A) \rightarrow H$ of the Steinberg group, and finally use the results of Section 2 to verify that $\tilde{\sigma}$ descends to an abstract representation of $E_{n}(A)$. Then, to conclude the argument, we will prove that this abstract representation is actually a morphism of algebraic groups.

We begin with the construction of the algebraic ring $A$ attached to a given representation $\rho$.

Proposition 3.3. Suppose $R$ is an associative ring, $K$ is an algebraically closed field, and $n \geq 3$. Given a representation $\rho: E_{n}(R) \rightarrow \mathrm{GL}_{m}(K)$, there exists an associative algebraic ring $A$ together with a homomorphism of abstract rings $f: R \rightarrow A$ having Zariski-dense image such that for all $i, j \in\{1, \ldots, n\}, i \neq j$, there is an injective regular map $\psi_{i j}: A \rightarrow H$ into $H:=\overline{\rho\left(E_{n}(R)\right)}$ satisfying

$$
\rho\left(e_{i j}(t)\right)=\psi_{i j}(f(t))
$$

for all $t \in R$. 
Proof. This statement goes back to [Kassabov and Sapir 2009] (see also [Rapinchuk 2011, Theorem 3.1]). For the sake of completeness, we indicate the main points of the construction. Let $A=\overline{\rho\left(e_{13}(R)\right)}$. If $\boldsymbol{\alpha}: A \times A \rightarrow A$ denotes the restriction of the matrix product in $H$ to $A$, it is clear $(A, \boldsymbol{\alpha})$ is a commutative algebraic subgroup of $H$. We let $f: R \rightarrow A$ be the map defined by $t \mapsto \rho\left(e_{13}(t)\right)$. From the definition, it follows that

$$
\boldsymbol{\alpha}\left(f\left(t_{1}\right), f\left(t_{2}\right)\right)=f\left(t_{1}+t_{2}\right)
$$

for all $t_{1}, t_{2} \in R$. To define the multiplication operation $\mu: A \times A \rightarrow A$, we will need the elements

$$
\bar{w}_{12}=e_{12}(1) e_{21}(-1) e_{12}(1) \quad \text { and } \quad \bar{w}_{23}=e_{23}(1) e_{32}(-1) e_{23}(1)
$$

(notice that these are simply the images under $\pi_{R}$ of the elements $w_{i j}(1)$ considered in Section 2). By direct computation, one sees that

$$
\bar{w}_{12}^{-1} e_{13}(r) \bar{w}_{12}=e_{23}(r), \quad \bar{w}_{23} e_{13}(r) \bar{w}_{23}^{-1}=e_{12}(r),
$$

and

$$
\left[e_{12}(r), e_{23}(s)\right]=e_{13}(r s)
$$

for all $r, s \in R$, where $[g, h]=g h g^{-1} h^{-1}$. Now let $\mu: A \times A \rightarrow H$ be the regular map defined by

$$
\boldsymbol{\mu}\left(a_{1}, a_{2}\right)=\left[\rho\left(\bar{w}_{23}\right) a_{1} \rho\left(\bar{w}_{23}\right)^{-1}, \rho\left(\bar{w}_{12}\right)^{-1} a_{2} \rho\left(\bar{w}_{12}\right)\right] .
$$

Then the above relations yield

$$
\boldsymbol{\mu}\left(f\left(t_{1}\right), f\left(t_{2}\right)\right)=f\left(t_{1} t_{2}\right),
$$

so, in particular, $\boldsymbol{\mu}(f(R) \times f(R)) \subset f(R)$, which implies that $\boldsymbol{\mu}(A \times A) \subset A$ and allows us to view $\boldsymbol{\mu}$ as a regular map $\boldsymbol{\mu}: A \times A \rightarrow A$. Since by our assumption $R$ is a (unital) associative ring and $f$ has Zariski-dense image, it follows that $(A, \boldsymbol{\alpha}, \boldsymbol{\mu})$ is a (unital) associative algebraic ring as defined in [Rapinchuk 2011, §2]. Furthermore, by our construction, (5) obviously holds for the inclusion map $\psi_{13}: A \rightarrow H$. Finally, using an appropriate element $\bar{w}_{i j}$, we can conjugate any root subgroup $e_{i j}(R)$ into $e_{13}(R)$, from which the existence of all the other maps $\psi_{i j}$ follows.

Remark 3.4. Observe that if $R$ is an infinite division ring, then the algebraic ring $A$ constructed in Proposition 3.3 is automatically connected. Indeed, the connected component $A^{\circ}$ is easily seen to be a two-sided ideal of $A$. So if $A \neq A^{\circ}$, then $f^{-1}\left(A^{\circ}\right)$ would be a proper two-sided ideal of finite index in $R$, which is impossible. In particular, we see that in the situation of Theorem 3.1, the algebraic ring associated to $\rho$ is connected. 
Next, we show that the representation $\rho$ can be lifted to a representation of the Steinberg group $\operatorname{St}_{n}(A)$. The precise statement is given by the following proposition:

Proposition 3.5. Suppose $R$ is an associative ring, $K$ is an algebraically closed field, and $n \geq 3$, and let $\rho: E_{n}(R) \rightarrow \mathrm{GL}_{m}(K)$ be a representation. Let $A$ and $f: R \rightarrow A$ be the algebraic ring and ring homomorphism constructed in Proposition 3.3. Then there exists a group homomorphism $\tilde{\tau}: \operatorname{St}_{n}(A) \rightarrow H \subset \mathrm{GL}_{m}(K)$ such that $\tilde{\tau}: x_{i j}(a) \mapsto \psi_{i j}(a)$ for all $a \in A$ and all $i, j \in\{1, \ldots, n\}, i \neq j$. Consequently, $\tilde{\tau} \circ \tilde{F}=\rho \circ \pi_{R}$, where $\tilde{F}: \operatorname{St}_{n}(R) \rightarrow \operatorname{St}_{n}(A)$ is the homomorphism induced by $f$.

Proof. This proposition is proved in exactly the same way as [Rapinchuk 2011,

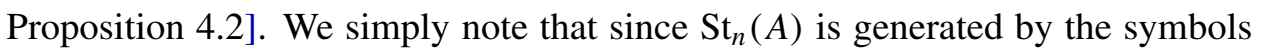
$x_{i j}(a)$ subject to the relations (R1)-(R3) given in Section 2, to establish the existence of $\tilde{\tau}$, it suffices to verify that relations (R1)-(R3) are satisfied if the $x_{i j}(a)$ are replaced by $\psi_{i j}(a)$, which follows from (5) and the fact that $f$ has Zariski-dense image. For the second statement, we observe that the maps $\tilde{\tau} \circ \tilde{F}$ and $\rho \circ \pi_{R}$ both send the symbol $x_{i j}(s)$ to $\psi_{i j}(f(s))=\rho\left(e_{i j}(s)\right)=\left(\rho \circ \pi_{R}\right)\left(x_{i j}(s)\right)$, so they must coincide on $\mathrm{St}_{n}(R)$.

To analyze the representation $\tilde{\sigma}$ that we have just constructed, we will need some additional information on the structure of the group $\mathrm{St}_{n}(A)$.

Proposition 3.6. Let $K$ be an algebraically closed field of characteristic 0 and $n$ an integer $\geq 3$. Suppose $A$ is an associative algebraic ring over $K$ such that $2 \in A^{\times}$, and let $A^{\circ}$ denote the connected component of $0_{A}$. Then

(i) $\operatorname{St}_{n}(A)=\operatorname{St}_{n}\left(A^{\circ}\right) \times P$, where $P$ is a finite group and

(ii) $K_{2}\left(n, A^{\circ}\right)$ is a central subgroup of $\mathrm{St}_{n}\left(A^{\circ}\right)$.

Proof. (i) First, since char $K=0$, by [Rapinchuk 2011, Proposition 2.14], we have $A=A^{\circ} \oplus S$ with $S$ a finite ring. So

$$
\mathrm{St}_{n}(A)=\mathrm{St}_{n}\left(A^{\circ}\right) \times \mathrm{St}_{n}(S),
$$

and we need to show that $\operatorname{St}_{n}(S)$ is a finite group. Now, since $E_{n}(S)$ is obviously a finite group and $K_{2}(n, S)$ is by definition the kernel of the canonical map $\pi_{S}: \operatorname{St}_{n}(S) \rightarrow E_{n}(S)$, we see that the finiteness of $\operatorname{St}_{n}(S)$ is equivalent to that of $K_{2}(n, S)$. On the other hand, since $2 \in S^{\times}$, Proposition 2.3 implies that $K_{2}(n, S)$ is a central subgroup of $\operatorname{St}_{n}(S)$. So we can use the argument given in the proof of [Rapinchuk 2011, Proposition 4.5] and consider the Hochschild-Serre spectral sequence

$$
H^{1}\left(\mathrm{St}_{n}(S), \mathbb{Q} / \mathbb{Z}\right) \rightarrow H^{1}\left(K_{2}(\Phi, S), \mathbb{Q} / \mathbb{Z}\right)^{\mathrm{St}_{n}(S)} \rightarrow H^{2}\left(E_{n}(S), \mathbb{Q} / \mathbb{Z}\right)
$$


(where all groups act trivially on $\mathbb{Q} / \mathbb{Z}$ ) corresponding to the short exact sequence

$$
1 \rightarrow K_{2}(n, S) \rightarrow \mathrm{St}_{n}(S) \stackrel{\pi_{S}}{\longrightarrow} E_{n}(S) \rightarrow 1
$$

to conclude that $K_{2}(n, S)$ is finite.

(ii) By [Rapinchuk 2011, Proposition 2.14], $A^{\circ}$ is a finite-dimensional $K$-algebra, so the assertion follows from Proposition 2.3.

Remark 3.7. We would like to point out that the assumption that $2 \in A^{\times}$is needed to guarantee that the finite ring $S$ that appears in the proof of Proposition 3.6(i) above is additively generated by its units, which then enables us to use Proposition 2.3 to conclude that $K_{2}(n, S)$ is a central subgroup of $\operatorname{St}_{n}(S)$. If $S$ is a finite commutative ring, then, as we show in [Rapinchuk 2011, Proposition 4.5], this assumption is not needed since in that case $S$ can be written as a finite product of commutative local rings, which are automatically generated by their units.

To complete the proofs of Theorems 3.1 and 3.2, the basic idea will be to show that the homomorphism $\tilde{\tau}$ constructed in Proposition 3.5 descends to a (rational) representation of $E_{n}(A)$. Let us make this more precise. Given a representation $\rho: E_{n}(R) \rightarrow \mathrm{GL}_{m}(K)$, let $f: R \rightarrow A$ be the ring homomorphism associated to $\rho$ (Proposition 3.3), and let $\tilde{F}: \mathrm{St}_{n}(R) \rightarrow \mathrm{St}_{n}(A)$ and $F: E_{n}(R) \rightarrow E_{n}(A)$ denote the group homomorphisms induced by $f$. Then under the hypotheses of Theorems 3.1 and 3.2, we have $\operatorname{St}_{n}(A)=\operatorname{St}_{n}\left(A^{\circ}\right)\left(\right.$ Remark 3.4) and $\mathrm{St}_{n}(A)=\operatorname{St}_{n}\left(A^{\circ}\right) \times P$ (Proposition 3.6), respectively, so in both cases, $\tilde{\Delta}:=\tilde{F}^{-1}\left(\operatorname{St}_{n}\left(A^{\circ}\right)\right)$ and $\Delta:=\pi_{R}(\tilde{\Delta})$ are finite-index subgroups of $\operatorname{St}_{n}(R)$ and $E_{n}(R)$. Moreover, $F(\Delta) \subset E_{n}\left(A^{\circ}\right)$ clearly. Thus, letting $\tilde{\sigma}$ denote the restriction of $\tilde{\tau}$ to $\operatorname{St}_{n}\left(A^{\circ}\right)$, we see that the solid arrows in

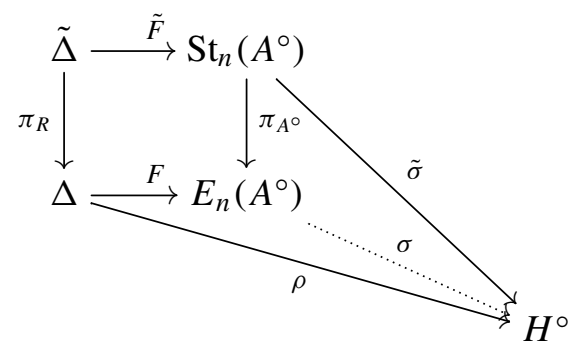

form a commutative diagram. In the remainder of this section, we will show that under our assumptions, there exists a group homomorphism $\sigma: E_{n}\left(A^{\circ}\right) \rightarrow H^{\circ}$ (in fact, a morphism of algebraic groups) making the full diagram commute. In the situation of Theorem 3.1, the existence of the required abstract homomorphism $\sigma$ will be shown in Proposition 3.8 below. For Theorem 3.2, we will first need to establish the somewhat weaker result that there exists a homomorphism $\bar{\sigma}: E_{n}\left(A^{\circ}\right) \rightarrow \bar{H}$ such that $\bar{\sigma} \circ \pi_{A^{\circ}}=v \circ \tilde{\sigma}$, where $Z\left(H^{\circ}\right)$ is the center of $H^{\circ}, \bar{H}=H^{\circ} / Z\left(H^{\circ}\right)$, and $v: H^{\circ} \rightarrow \bar{H}$ is the canonical map (see Proposition 3.10). 
Proposition 3.8. Suppose $k$ and $K$ are fields of characteristic 0 with $K$ algebraically closed, $D$ is a finite-dimensional central division algebra over $k$, and $n$ is an integer $\geq 3$. Let $\rho: E_{n}(D) \rightarrow \mathrm{GL}_{m}(K)$ be a representation, and let $A$ denote the algebraic ring associated to $\rho$ (Proposition 3.3). Then $A=A^{\circ}$ is a finite-dimensional $K$-algebra and there exists a homomorphism of abstract groups $\sigma: E_{n}\left(A^{\circ}\right) \rightarrow H^{\circ}$ making the diagram (6) commute.

Proof. We have $A=A^{\circ}$ by Remark 3.4, and $A^{\circ}$ is a finite-dimensional $K$-algebra by [Rapinchuk 2011, Proposition 2.14]. Next, by Proposition 2.4, $K_{2}(n, A)$ coincides with the subgroup

$$
U_{n}^{\prime}(A)=\left\langle c(u, v) \mid u, v \in \overline{f\left(L^{\times}\right)}\right\rangle
$$

of $\operatorname{St}_{n}(A)$, where $L$ is an arbitrary maximal subfield of $D$ and $f: D \rightarrow A$ is the ring homomorphism associated to $\rho$. Now, from the construction of $\tilde{\sigma}$ and the definition of $c(u, v)$, we have

$$
\tilde{\sigma}(c(u, v))=H_{12}(u) H_{12}(v) H_{12}(v u)^{-1},
$$

where for $r \in A^{\times}$, we set

$$
H_{12}(r)=W_{12}(r) W_{12}(-1) \quad \text { and } \quad W_{12}(r)=\psi_{12}(r) \psi_{21}\left(-r^{-1}\right) \psi_{12}(r) .
$$

By [Rapinchuk 2011, Proposition 2.4], the map $A^{\times} \rightarrow A^{\times}, t \mapsto t^{-1}$ is regular, which implies that the map

$$
\Theta: A^{\times} \times A^{\times} \rightarrow H, \quad(u, v) \mapsto \tilde{\tau}(c(u, v))
$$

is also regular. On the other hand, as we observed earlier, $\pi_{D}\left(h_{i j}(u)\right) \in E_{n}(D)$ is a diagonal matrix with $u$ as the $i$-th diagonal entry, $u^{-1}$ as the $j$-th diagonal entry, and $1 \mathrm{~s}$ everywhere else on the diagonal. In particular, for $u, v \in L^{\times}$, it follows that

$$
\pi_{D}\left(h_{12}(u) h_{12}(v) h_{12}(v u)^{-1}\right)=1 .
$$

So by Proposition 3.5,

$$
\tilde{\sigma}(c(f(u), f(v)))=\rho\left(\pi_{D}\left(h_{12}(u) h_{12}(v) h_{12}(v u)^{-1}\right)\right)=1
$$

for all $u, v \in L^{\times}$. By the regularity of $\Theta$, we obtain that $\tilde{\sigma}(c(a, b))=1$ for all $a, b \in \overline{f\left(L^{\times}\right)}$, and consequently, $\tilde{\sigma}$ vanishes on $K_{2}(n, A)$. Since the canonical homomorphism $\pi_{A}: \operatorname{St}_{n}(A) \rightarrow E_{n}(A)$ is surjective and $K_{2}(n, A)=\operatorname{ker} \pi_{A}$ by definition, the existence of $\sigma$ now follows.

The proof of Theorem 3.2 will require the following proposition, which contains analogs of results established in [Rapinchuk 2011, §5]:

Proposition 3.9. Suppose $R$ is an associative ring with $2 \in R^{\times}, K$ is an algebraically closed field of characteristic 0 , and $n \geq 3$. Let $\rho: E_{n}(R) \rightarrow \mathrm{GL}_{m}(K)$ be a 
representation, set $H=\overline{\rho\left(E_{n}(R)\right)}$, and let A denote the algebraic ring associated to $\rho$. Then the following hold:

(i) The group $H^{\circ}$ coincides with $\tilde{\sigma}\left(\operatorname{St}_{n}\left(A^{\circ}\right)\right)$ and is its own commutator.

(ii) Let $U$ and $Z\left(H^{\circ}\right)$ be the unipotent radical and center of $H^{\circ}$, respectively. If $U$ is commutative, then $Z\left(H^{\circ}\right) \cap U=\{e\}$, and consequently, $Z\left(H^{\circ}\right)$ is finite and is contained in any Levi subgroup of $H^{\circ}$.

Proof. (i) It follows from Proposition 3.5 that $\tilde{\sigma}\left(\mathrm{St}_{n}\left(A^{\circ}\right)\right)$ coincides with the (abstract) group $\mathscr{H} \subset H$ generated by all the $\psi_{i j}\left(A^{\circ}\right)$ with $i, j \in\{1, \ldots, n\}, i \neq j$. Since $\psi_{\alpha}\left(A^{\circ}\right)$ is clearly a connected subgroup of $H$, by [Borel 1991, Proposition 2.2], $\mathscr{H}$ is Zariski-closed and connected; hence, $\mathscr{H} \subset H^{\circ}$. On the other hand, by Proposition 3.6, $\operatorname{St}_{n}\left(A^{\circ}\right)$ is a finite-index subgroup of $\operatorname{St}_{n}(A)$, from which it follows that $\tilde{\sigma}\left(\operatorname{St}_{n}(A)\right)$ is Zariski-closed. Since $\tilde{\sigma}\left(\operatorname{St}_{n}(A)\right)$ contains $\rho\left(E_{n}(R)\right)$, it is Zariski-dense in $H$ and therefore coincides with $H$. So $\mathscr{H}$ is a closed subgroup of finite index in $H$; hence, $\mathscr{H} \supset H^{\circ}$, and consequently, $\mathscr{H}=H^{\circ}$. Furthermore, from the definition of the Steinberg group, one easily sees that $\mathrm{St}_{n}\left(A^{\circ}\right)$ coincides with its commutator subgroup, so the same is true for $H^{\circ}$.

(ii) Using the fact that $H^{\circ}$ coincides with its commutator subgroup, one can now apply the argument given in the proof of [Rapinchuk 2011, Proposition 5.5].

Now set $\bar{H}=H^{\circ} / Z\left(H^{\circ}\right)$. Since $Z\left(H^{\circ}\right)$ is a closed normal subgroup of $H^{\circ}$, $\bar{H}$ is an (affine) algebraic group and the canonical map $v: H^{\circ} \rightarrow \bar{H}$ is a morphism of algebraic groups [Borel 1991, Theorem 6.8].

Proposition 3.10. Suppose $R$ is an associative ring with $2 \in R^{\times}, K$ is an algebraically closed field of characteristic 0 , and $n \geq 3$. Let $\rho: E_{n}(R) \rightarrow \mathrm{GL}_{m}(K)$ be a representation, set $H=\overline{\rho\left(E_{n}(R)\right)}$, and let $A$ denote the algebraic ring associated to $\rho$. Then $A^{\circ}$ is a finite-dimensional $K$-algebra and there exists a homomorphism $\bar{\sigma}: E_{n}\left(A^{\circ}\right) \rightarrow \bar{H}$ such that $\bar{\sigma} \circ \pi_{A^{\circ}}=v \circ \tilde{\sigma}$.

Proof. Since char $K=0$, by [Rapinchuk 2011, Proposition 2.14], $A^{\circ}$ is a finitedimensional $K$-algebra. Furthermore, $H^{\circ}=\tilde{\sigma}\left(\operatorname{St}_{n}\left(A^{\circ}\right)\right)$ by Proposition 3.9 and $K_{2}\left(n, A^{\circ}\right)=\operatorname{ker} \pi_{A^{\circ}}$ is a central subgroup of $\mathrm{St}_{n}\left(A^{\circ}\right)$ by Proposition 2.3, from which the existence of $\bar{\sigma}$ follows.

The remaining step in the proof is to show that the (abstract) homomorphisms $\sigma: E_{n}\left(A^{\circ}\right) \rightarrow H^{\circ}$ and $\bar{\sigma}: E_{n}\left(A^{\circ}\right) \rightarrow \bar{H}$ constructed in Propositions 3.8 and 3.10, respectively, are actually morphisms of algebraic groups (see Proposition 3.12 below). In the latter case, this will allow us to lift $\bar{\sigma}$ to a morphism of algebraic groups $\sigma: E_{n}\left(A^{\circ}\right) \rightarrow H^{\circ}$ making the diagram (6) commute. Our proof of rationality here will deviate from the approach of [Rapinchuk 2011] as rather than using results about the "big cell" of $E_{n}\left(A^{\circ}\right)$, we will instead apply the following geometric lemma: 
Lemma 3.11. Let $X, Y$, and $Z$ be irreducible varieties over an algebraically closed field $K$ of characteristic 0 . Suppose $s: X \rightarrow Y$ and $t: X \rightarrow Z$ are regular maps with $s$ dominant such that for any $x_{1}, x_{2} \in X$ with $s\left(x_{1}\right)=s\left(x_{2}\right)$, we have $t\left(x_{1}\right)=t\left(x_{2}\right)$. Then there exists a rational map $h: Y \rightarrow Z$ such that $h \circ s=t$ on a suitable open subset of $X$.

Proof. Let $W \subset X \times Y \times Z$ be the subset

$$
W=\{(x, y, z) \mid s(x)=y, t(x)=z\} .
$$

Notice that $W$ is the graph of the morphism

$$
\varphi: X \rightarrow Y \times Z, \quad x \mapsto(s(x), t(x)),
$$

so $W$ is an irreducible variety isomorphic to $X$. Now consider the projection $\operatorname{pr}_{Y \times Z}: X \times Y \times Z \rightarrow Y \times Z$, and let $U=\operatorname{pr}_{Y \times Z}(W)$ and $V=\bar{U}$, where the bar denotes the Zariski closure. Then $V$ is an irreducible variety. Moreover, $U$ is constructible by [Humphreys 1975, Theorem 4.4] so in particular contains a dense open subset $P$ of $V$, which is itself an irreducible variety. Let now $p: P \rightarrow Y$ be the projection to the first component. We claim that $p$ gives a birational isomorphism between $P$ and $Y$. From our assumptions, we see that $p$ is dominant, and since char $K=0, p$ is also separable. So it follows from [Humphreys 1975, Theorem 4.6] that to show that $p$ is birational, we only need to verify that it is injective. Consider $u_{1}=\left(y_{1}, z_{1}\right)$ and $u_{2}=\left(y_{2}, z_{2}\right)$ in $P$, with $y_{1}=y_{2}$. By our construction, there exist $x_{1}, x_{2} \in X$ such that $s\left(x_{1}\right)=y_{1}, t\left(x_{1}\right)=z_{1}, s\left(x_{2}\right)=y_{2}$, and $t\left(x_{2}\right)=z_{2}$. Since $s\left(x_{1}\right)=s\left(x_{2}\right)$, we have $t\left(x_{1}\right)=t\left(x_{2}\right)$, so $u_{1}=u_{2}$, as needed.

Since $p$ is birational, we can now take $h=\pi_{Z} \circ p^{-1}: Y \rightarrow Z$, where we let $\pi_{Z}: Y \times Z \rightarrow Z$ be the projection.

Now let $\rho: E_{n}(R) \rightarrow \mathrm{GL}_{m}(K)$ be a representation as in Theorem 3.1 or 3.2, and let $A$ denote the algebraic ring associated to $\rho$. Also let $Q$ be the set of all pairs $(i, j)$ with $1 \leq i, j \leq n, i \neq j$. Then, as we already observed at the beginning of this section, $E_{n}\left(A^{\circ}\right)$ is the connected algebraic group generated by the images $W_{q}=\operatorname{im} \varphi_{q}$ of the regular maps

$$
\varphi_{q}: A^{\circ} \rightarrow \mathrm{GL}_{n}\left(A^{\circ}\right), \quad a \mapsto e_{q}(a)
$$

for all $q \in Q$. In particular, [Borel 1991, Proposition 2.2] implies that there exists a finite sequence $(\alpha(1), \ldots, \alpha(v))$ in $Q$ such that

$$
E_{n}\left(A^{\circ}\right)=W_{\alpha(1)}^{\varepsilon_{1}} \cdots W_{\alpha(v)}^{\varepsilon_{v}},
$$

where each $\varepsilon_{i}= \pm 1$. Let

$$
X=\prod_{i=1}^{v}\left(A^{\circ}\right)_{\alpha(i)}
$$


be the product of $v$ copies of $A^{\circ}$ indexed by the $\alpha(i)$, and define a regular map $s: X \rightarrow E_{n}\left(A^{\circ}\right)$ by

$$
s\left(a_{\alpha(1)}, \ldots, a_{\alpha(v)}\right)=\varphi_{\alpha(1)}\left(a_{\alpha(1)}\right)^{\varepsilon_{1}} \cdots \varphi_{\alpha(v)}\left(a_{\alpha(v)}\right)^{\varepsilon_{v}} .
$$

Also let

$$
t: X \rightarrow H^{\circ}, \quad t\left(a_{\alpha(1)}, \ldots, a_{\alpha(v)}\right)=\psi_{\alpha(1)}\left(a_{\alpha(1)}\right)^{\varepsilon_{1}} \cdots \psi_{\alpha(v)}\left(a_{\alpha(v)}\right)^{\varepsilon_{v}},
$$

where the $\psi_{\alpha(i)}$ are the regular maps from Proposition 3.3. With this setup, we can now prove:

Proposition 3.12. The homomorphisms $\sigma: E_{n}\left(A^{\circ}\right) \rightarrow H^{\circ}$ and $\bar{\sigma}: E_{n}\left(A^{\circ}\right) \rightarrow \bar{H}$ constructed in Propositions 3.8 and 3.10, respectively, are morphisms of algebraic groups.

Proof. We will only consider $\sigma$ as the argument for $\bar{\sigma}$ is completely analogous. Set $Y=E_{n}\left(A^{\circ}\right)$ and $Z=H^{\circ}$, and let $s: X \rightarrow Y$ and $t: X \rightarrow Z$ be the regular maps defined in (7) and (8). From the construction of $\sigma$, it is clear that $(\sigma \circ s)(x)=t(x)$, so in particular, $s\left(x_{1}\right)=s\left(x_{2}\right)$ for $x_{1}, x_{2} \in X$ implies that $t\left(x_{1}\right)=t\left(x_{2}\right)$. Hence, by Lemma 3.11, $\sigma$ is a rational map. Therefore, there exists an open subset $V \subset E_{n}\left(A^{\circ}\right)$ such that $\left.\sigma\right|_{V}$ is regular. So it follows from the next lemma that $\sigma: E_{n}\left(A^{\circ}\right) \rightarrow H^{\circ}$ is a morphism.

Lemma 3.13 [Rapinchuk 2011, Lemma 6.4]. Let $K$ be an algebraically closed field, and let $\varphi$ and $\varphi^{\prime}$ be affine algebraic groups over $K$ with $\varphi$ connected. Suppose $f: G \rightarrow \mathcal{G}^{\prime}$ is an abstract group homomorphism, ${ }^{2}$ and assume there exists a Zariskiopen set $V \subset G$ such that $\varphi:=\left.f\right|_{V}$ is a regular map. Then $f$ is a morphism of algebraic groups.

Theorem 3.1 now follows from Propositions 3.8 and 3.12 with $\mathscr{B}=A^{\circ}(=A)$. For Theorem 3.2, we again take $\mathscr{B}=A^{\circ}$, and it remains to show that one can lift the morphism $\bar{\sigma}: E_{n}\left(A^{\circ}\right) \rightarrow \bar{H}$ to a morphism $\sigma: E_{n}\left(A^{\circ}\right) \rightarrow H^{\circ}$ making the diagram (6) commute. This is accomplished through a suitable modification of the argument used in the proof of [Rapinchuk 2011, Proposition 6.6]. For this, we need some analogs of results contained in [Rapinchuk 2011, §6] regarding the structure of $E_{n}(B)$ as an algebraic $K$-group, where $B$ is an arbitrary finite-dimensional algebra over an algebraically closed field $K$. Let $J=J(B)$ be the Jacobson radical of $B$. Then by the Wedderburn-Malcev theorem [Pierce 1982, Corollary 11.6], there exists a semisimple subalgebra $\bar{B} \subset B$ such that $B=\bar{B} \oplus J$ as $K$-vector spaces and $\bar{B} \simeq B / J$ as $K$-algebras. Furthermore, since $K$ is algebraically closed, the Artin-Wedderburn theorem implies that

$$
\bar{B}=M_{n_{1}}(K) \times \cdots \times M_{n_{r}}(K) .
$$

${ }^{2}$ Here we tacitly identify $\mathscr{G}$ and $\mathscr{G}^{\prime}$ with the corresponding groups $\mathscr{G}(K)$ and $\mathscr{G}^{\prime}(K)$ of $K$-points. 
Now consider the group homomorphism $E_{n}(B) \rightarrow E_{n}(\bar{B})$ induced by the canonical map $B \rightarrow B / J$ (notice that this is a morphism of algebraic groups as $B \rightarrow B / J$ is a homomorphism of algebraic rings: see [Rapinchuk 2011, Lemma 2.9]), and let $E_{n}(J)$ be its kernel. It is clear that $E_{n}(J)$ is a closed normal subgroup of $E_{n}(B)$. Note that

$$
E_{n}\left(M_{n_{i}}(K)\right) \simeq E_{n n_{i}}(K) \simeq \mathrm{SL}_{n n_{i}}(K),
$$

so $E_{n}(\bar{B})$ is a semisimple simply connected algebraic group. It is also easy to see that for any $a, b \geq 1$, we have

$$
\left[\mathrm{GL}_{n}\left(B, J^{a}\right), \mathrm{GL}_{n}\left(B, J^{b}\right)\right] \subset \mathrm{GL}_{n}\left(B, J^{a+b}\right),
$$

where $\mathrm{GL}_{n}\left(B, J^{s}\right)=\operatorname{ker}\left(\mathrm{GL}_{n}(B) \rightarrow \mathrm{GL}_{n}\left(B / J^{s}\right)\right)$. Since $J$ is a nilpotent ideal, it follows that $E_{n}(J)$ is a nilpotent group. In particular, we obtain that

$$
E_{n}(B)=E_{n}(J) \rtimes E(\bar{B})
$$

is a Levi decomposition of $E_{n}(B)$ [Rapinchuk 2011, Proposition 6.5].

Now, using the Levi decomposition (9) for $B=\mathscr{B}$ as well as the fact that the center $Z\left(H^{\circ}\right)$ is finite (Proposition 3.9), one can directly imitate the argument of [Rapinchuk 2011, Proposition 6.6] to conclude the proof of Theorem 3.2.

Finally, to derive Theorem 1 from Theorem 3.1, we first note that by Lemma 2.5, we have $K$-algebra isomorphisms

$$
\mathscr{B} \simeq D \otimes_{k} C \simeq M_{s}(C),
$$

where $s^{2}=\operatorname{dim}_{k} D$ and $C=\overline{f(k)}$ (as above, $f: D \rightarrow \mathscr{R}$ is the ring homomorphism associated to $\rho)$. Consequently, $E_{n}(\mathscr{B}) \simeq E_{n}\left(M_{s}(C)\right) \simeq E_{n s}(C)$. Moreover, since $C$ is a finite-dimensional $K$-algebra, in particular a semilocal commutative ring, $E_{n s}(C) \simeq \mathrm{SL}_{n s}(C)$ [Matsumoto 1966, Corollary 2]. So since $G=\mathbf{S L}_{n, D}$ is $K$ isomorphic to $\mathrm{SL}_{n s}$ [Platonov and Rapinchuk 1994, 2.3.1], we see $E_{n}(\mathscr{B}) \simeq G(C)$. Letting $f_{C}: k \rightarrow C$ be the restriction of $f$ to $k$, we now obtain Theorem 1 .

\section{Applications to representation varieties and deformations of representations}

In this section, we will prove Theorem 2. To estimate the dimension of the character variety $X_{n}(\Gamma)$ for an elementary subgroup $\Gamma$ as in the statement of Theorem 2, we will exploit the well known connection, going back to A. Weil, between the tangent space of $X_{n}(\Gamma)$ at a given point and the 1-cohomology of $\Gamma$ with coefficients in the space of a naturally associated representation. We then use the results of [Rapinchuk 2011] on standard descriptions of representations of $\Gamma$ to relate the latter space to a certain space of derivations of the finitely generated ring $R$ used to define $\Gamma$ (see Proposition 4.4). Since the dimensions of spaces of derivations are finite and 
are bounded by a constant depending only on $R$, we obtain the required bound on $\operatorname{dim} X_{n}(\Gamma)$. Throughout this section, we will work over a fixed algebraically closed field $K$ of characteristic 0 .

We begin by summarizing some key definitions and basic properties related to representation and character varieties, mostly following the first two chapters of [Lubotzky and Magid 1985]. Let $\Gamma$ be a finitely generated group, and fix an integer $n \geq 1$. Recall that the $n$-th representation scheme of $\Gamma$ is the functor $\Re_{n}(\Gamma)$ from the category of commutative $K$-algebras to the category of sets defined by

$$
\mathfrak{R}_{n}(\Gamma)(A)=\operatorname{Hom}\left(\Gamma, \mathrm{GL}_{n}(A)\right) .
$$

More generally, if $\varphi$ is a linear algebraic group over $K$, we let the representation scheme of $\Gamma$ with values in $\mathscr{G}$ be the functor $\mathfrak{R}(\Gamma, \mathscr{G})$ defined by

$$
\mathfrak{R}(\Gamma, \mathscr{G})(A)=\operatorname{Hom}(\Gamma, \mathscr{G}(A)) .
$$

Because for any commutative $K$-algebra $A$, a homomorphism $\rho: \Gamma \rightarrow \mathrm{GL}_{n}(A)$ is determined by the images of the generators, subject to the defining relations of $\Gamma$, one shows that $\mathfrak{R}_{n}(\Gamma)$ is an affine $K$-scheme represented by a finitely generated $K$-algebra $\mathfrak{A}_{n}(\Gamma)$. Similarly, $\mathfrak{R}(\Gamma, \mathscr{G})$ is an affine $K$-scheme represented by a finitely generated $K$-algebra $\mathfrak{A}(\Gamma, \mathscr{G})$ [Lubotzky and Magid 1985, Proposition 1.2]. The set $\mathfrak{R}_{n}(\Gamma)(K)$ of $K$-points of $\mathfrak{R}_{n}(\Gamma)$ is then denoted $R_{n}(\Gamma)$ and is called the $n$-th representation variety of $\Gamma$. It is an affine variety over $K$ with coordinate ring $A_{n}(\Gamma)=\mathfrak{A}_{n}(\Gamma)_{\text {red }}$, the quotient of $\mathfrak{A}_{n}(\Gamma)$ by its nilradical. The representation variety $R(\Gamma, \mathscr{G})$ is defined analogously.

Now let $\rho_{0} \in R(\Gamma, \mathscr{G})$. To describe the Zariski tangent space of $\mathfrak{R}(\Gamma, \mathscr{G})$ at $\rho_{0}$, denoted $T_{\rho_{0}}(\Re(\Gamma, \mathscr{G})$ ), we will use the algebra of dual numbers $K[\varepsilon]$ (where $\left.\varepsilon^{2}=0\right)$. More specifically, it is well known that $\mathfrak{R}(\Gamma, \mathscr{G})(K[\varepsilon])$ is the tangent bundle of $\mathfrak{R}(\Gamma, \mathscr{G})$, and therefore, $T_{\rho_{0}}(\mathfrak{R}(\Gamma, \mathscr{G}))$ can be identified with the fiber over $\rho_{0}$ of the map $\mu: \mathfrak{R}(\Gamma, \mathscr{G})(K[\varepsilon]) \rightarrow \mathfrak{R}(\Gamma, \mathscr{G})(K)$ induced by the augmentation homomorphism $K[\varepsilon] \rightarrow K, \varepsilon \mapsto 0$ [Borel 1991, AG 16.2]. In other words, we have

$$
T_{\rho_{0}}(\mathfrak{R}(\Gamma, \mathscr{G}))=\left\{\rho \in \operatorname{Hom}(\Gamma, \mathscr{G}(K[\varepsilon])) \mid \mu \circ \rho=\rho_{0}\right\} .
$$

For us, it will be useful to have the following alternative description of $T_{\rho_{0}}(\Re(\Gamma, \mathscr{G}))$. Let $\tilde{\mathfrak{g}}$ be the Lie algebra of $\mathscr{G}$. Notice that $\tilde{\mathfrak{g}}$ has a natural $\Gamma$-action given by

$$
\gamma \cdot x=\operatorname{Ad}\left(\rho_{0}(\gamma)\right) x
$$

for $\gamma \in \Gamma$ and $x \in \tilde{\mathfrak{g}}$, where Ad: $\mathscr{G}(K) \rightarrow \mathrm{GL}(\tilde{\mathfrak{g}})$ is the adjoint representation. Now $T_{\rho_{0}}(\Re(\Gamma, \mathscr{G}))$ can be identified with the space $Z^{1}(\Gamma, \tilde{\mathfrak{g}})$ of 1-cocycles [Lubotzky and Magid 1985, Proposition 2.2]. Indeed, an element $c \in Z^{1}(\Gamma, \tilde{\mathfrak{g}})$ is by definition 
a map $c: \Gamma \rightarrow \tilde{\mathfrak{g}}$ such that

$$
c\left(\gamma_{1} \gamma_{2}\right)=c\left(\gamma_{1}\right)+\operatorname{Ad}\left(\rho_{0}\left(\gamma_{1}\right)\right) c\left(\gamma_{2}\right) .
$$

On the other hand, we have an isomorphism $\mathscr{G}(K[\varepsilon]) \simeq \tilde{\mathfrak{g}} \rtimes \mathscr{G}$ given by

$$
B+C \varepsilon \mapsto\left(C B^{-1}, B\right) .
$$

Hence, an element $\rho \in T_{\rho_{0}}(\mathfrak{R}(\Gamma, \mathscr{G}))$ is a homomorphism $\rho: \Gamma \rightarrow \tilde{\mathfrak{g}} \rtimes \mathscr{G}$ whose projection to the second factor is $\rho_{0}$. In other words, it arises from a map $c: \Gamma \rightarrow \tilde{\mathfrak{g}}$ such that the map

$$
\Gamma \rightarrow \tilde{\mathfrak{g}} \rtimes \mathscr{G}, \quad \gamma \mapsto\left(c(\gamma), \rho_{0}(\gamma)\right)
$$

is a group homomorphism. With the above identification, this translates into the condition

$$
c\left(\gamma_{1} \gamma_{2}\right)=c\left(\gamma_{1}\right)+\operatorname{Ad}\left(\rho_{0}\left(\gamma_{1}\right)\right) c\left(\gamma_{2}\right) \text {, }
$$

giving the required isomorphism of $T_{\rho_{0}}(\Re(\Gamma, \mathscr{G}))$ with $Z^{1}(\Gamma, \tilde{\mathfrak{g}})$. Also notice that for any finite-index subgroup $\Delta \subset \Gamma$ (which is automatically finitely generated), the natural restriction maps $\mathfrak{R}(\Gamma, \mathscr{G}) \rightarrow \mathfrak{R}(\Delta, \mathscr{G})$ and $Z^{1}(\Gamma, \tilde{\mathfrak{g}}) \rightarrow Z^{1}(\Delta, \tilde{\mathfrak{g}})$ induce a commutative diagram

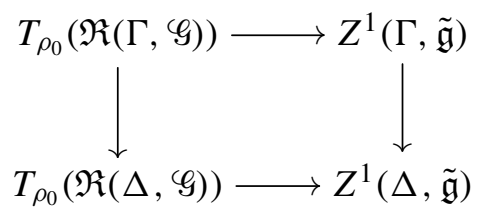

where the horizontal maps are the isomorphisms described above.

Next, let us recall a characterization of the space $B^{1}(\Gamma, \tilde{\mathfrak{g}})$ of 1 -coboundaries that will be used later; for this, we need to consider the action of $\mathscr{G}(K)$ on $R(\Gamma, \mathscr{G})$. Given $\rho_{0} \in R(\Gamma, \mathscr{G})$, let $\psi_{\rho_{0}}: \mathscr{G}(K) \rightarrow R(\Gamma, \mathscr{G})$ be the orbit map, i.e., the map defined by

$$
\psi_{\rho_{0}}(T)=T \rho_{0} T^{-1}, \quad T \in \mathscr{G}(K) .
$$

Direct computation shows that under the isomorphism $T_{\rho_{0}}(\Re(\Gamma, \mathscr{G})) \simeq Z^{1}(\Gamma, \tilde{\mathfrak{g}})$, the image of the differential $\left(d \psi_{\rho_{0}}\right)_{e}: T_{e}(\mathscr{G}) \rightarrow T_{\rho_{0}}(R(\Gamma, \mathscr{G})) \subset T_{\rho_{0}}(\Re(\Gamma, \mathscr{G}))$ consists of maps $\tau: \Gamma \rightarrow \tilde{\mathfrak{g}}$ such that there exists $A \in \tilde{\mathfrak{g}}$ with

$$
\tau(\gamma)=A-\operatorname{Ad}\left(\rho_{0}(\gamma)\right) A
$$

for all $\gamma \in \Gamma$, i.e., the image coincides with $B^{1}(\Gamma, \tilde{\mathfrak{g}})$ [Lubotzky and Magid 1985, Proposition 2.3]. In fact, if $O\left(\rho_{0}\right)$ is the orbit of $\rho_{0}$ in $R(\Gamma, \mathscr{G})$ under the action of $\mathscr{G}(K)$, then $B^{1}(\Gamma, \tilde{\mathfrak{g}})$ can be identified with $T_{\rho_{0}}\left(O\left(\rho_{0}\right)\right) \subset T_{\rho_{0}}(R(\Gamma, \mathscr{G}))$ [Lubotzky and Magid 1985, Corollary 2.4]. 
As a special case of the preceding constructions, we can consider the action of $\mathrm{GL}_{n}(K)$ on $R_{n}(\Gamma)$. The $n$-th character variety of $\Gamma$, denoted $X_{n}(\Gamma)$, is by definition the (categorical) quotient of $R_{n}(\Gamma)$ by $\mathrm{GL}_{n}(K)$; i.e., it is the affine $K$ variety with coordinate ring $A_{n}(\Gamma)^{\mathrm{GL}_{n}(K)}$. Let $\pi: R_{n}(\Gamma) \rightarrow X_{n}(\Gamma)$ be the canonical map. Then each fiber $\pi^{-1}(x)$ contains a semisimple representation, and moreover, if $\rho_{1}, \rho_{2} \in R_{n}(\Gamma)$ are semisimple with $\pi\left(\rho_{1}\right)=\pi\left(\rho_{2}\right)$, then $\rho_{1}=T \rho_{2} T^{-1}$ for some $T \in \mathrm{GL}_{n}(K)$. In particular, we see that $\pi$ induces a bijection between the isomorphism classes of semisimple representations and the points of $X_{n}(\Gamma)$ [Lubotzky and Magid 1985, Theorem 1.28].

We turn to the proof of Theorem 2. In the remainder of this section, $\Gamma$ will be the elementary subgroup $E(\Phi, R) \subset G(R)$, where $\Phi$ is a reduced irreducible root system of rank $\geq 2, G$ a universal Chevalley-Demazure group scheme of type $\Phi$, and $R$ a finitely generated commutative ring such that $(\Phi, R)$ is a nice pair. By recent work of Ershov, Jaikin-Zapirain, and Kassabov [2011], it is known that $\Gamma$ has Kazhdan's property $(\mathrm{T})$. In particular, $\Gamma$ is finitely generated and satisfies the condition

for any finite-index subgroup $\Delta \subset \Gamma$, the abelianization $\Delta^{\mathrm{ab}}=\Delta /[\Delta, \Delta]$ is finite

[de la Harpe and Valette 1989]. This has the following consequence:

Proposition 4.1 [Rapinchuk 1999, Proposition 2]. Let $\Gamma$ be a group satisfying (FAb). For any $n \geq 1$, there exists a finite collection $G_{1}, \ldots, G_{d}$ of algebraic subgroups of $\mathrm{GL}_{n}(K)$ such that for any completely reducible representation $\rho: \Gamma \rightarrow \mathrm{GL}_{n}(K)$, the Zariski closure $\overline{\rho(\Gamma)}$ is conjugate to one of the $G_{i}$. Moreover, for each $i$, the connected component $G_{i}^{\circ}$ is a semisimple group.

Thus, if we denote by $R_{n}(\Gamma)_{s s}$ the set of completely reducible representations $\rho: \Gamma \rightarrow \mathrm{GL}_{n}(K)$, we have ${ }^{3}$

$$
R_{n}(\Gamma)_{s s}=\bigcup_{\substack{i \in\{1, \ldots, d\}, g \in \mathrm{GL}_{n}(K)}} g R^{\prime}\left(\Gamma, G_{i}\right) g^{-1},
$$

where for an algebraic subgroup $\mathscr{G} \subset \mathrm{GL}_{n}(K)$, we set

$$
R^{\prime}(\Gamma, \mathscr{G})=\{\rho: \Gamma \rightarrow \mathscr{G} \mid \overline{\rho(\Gamma)}=\mathscr{G}\} .
$$

Therefore, letting $\pi: R_{n}(\Gamma) \rightarrow X_{n}(\Gamma)$ be the canonical map, we obtain that

$$
X_{n}(\Gamma)=\bigcup_{i=1}^{d} \pi\left(R^{\prime}\left(\Gamma, G_{i}\right)\right) .
$$

${ }^{3}$ Observe that if $\mathscr{G} \subset \mathrm{GL}_{n}(K)$ is an algebraic subgroup such that $\mathscr{G}^{\circ}$ is semisimple, then $\mathscr{G}$ is completely reducible; hence, any representation $\rho: \Gamma \rightarrow \mathrm{GL}_{n}(K)$ with $\overline{\rho(\Gamma)}=\mathscr{G}$ is completely reducible. 
Notice that if $\varphi \subset \mathrm{GL}_{n}(K)$ is an algebraic group such that $\mathscr{G}^{\circ}$ is semisimple, then $R^{\prime}(\Gamma, \mathscr{G})$ is an open subvariety of $R(\Gamma, \mathscr{G})$. Indeed, let

$$
R^{\prime \prime}(\Gamma, \mathscr{G})=\left\{\rho: \Gamma \rightarrow \mathscr{G} \mid \overline{\rho(\Gamma)} \supset \mathscr{G}^{\circ}\right\} .
$$

Since $\mathscr{G}^{\circ}$ is semisimple, $R^{\prime \prime}(\Gamma, \mathscr{G})$ is easily seen to be an open subvariety in $R(\Gamma, \mathscr{G})$ [Rapinchuk 1998, Lemma 4]. On the other hand, we obviously have

$$
R^{\prime}(\Gamma, \mathscr{G})=R^{\prime \prime}(\Gamma, \mathscr{G}) \cap\left(R(\Gamma, \mathscr{G}) \backslash \bigcup_{i=1}^{l} R\left(\Gamma, \mathscr{H}_{i}\right)\right),
$$

where $\mathscr{H}_{1}, \ldots, \mathscr{H}_{l}$ are the algebraic subgroups of $\mathscr{G}_{\text {such that }}$

$$
\mathscr{G} \supsetneq \mathscr{H}_{i} \supset \mathscr{G}^{\circ} .
$$

Now let $W \subset X_{n}(\Gamma)$ be an irreducible component of maximal dimension so that $\operatorname{dim} X_{n}(\Gamma)=\operatorname{dim} W$. Then it follows from (10) that we can find an irreducible component $V$ of some $R^{\prime}\left(\Gamma, G_{i}\right)$ such that $\overline{\pi(V)}=W$. Since $\left.\pi\right|_{V}$ is dominant and separable (as char $K=0$ ), it follows from [Borel 1991, AG 17.3] that there exists $\rho_{0} \in V$ that is a simple point (of $R^{\prime}\left(\Gamma, G_{i}\right)$ ) such that $\pi\left(\rho_{0}\right)$ is simple and the differential

$$
(d \pi)_{\rho_{0}}: T_{\rho_{0}}(V) \rightarrow T_{\pi\left(\rho_{0}\right)}(W)
$$

is surjective. Next, let $\psi_{\rho_{0}}: G_{i} \rightarrow R\left(\Gamma, G_{i}\right)$ be the orbit map. By the construction of $\pi$, we have $\left(\pi \circ \psi_{\rho_{0}}\right)(T)=\pi\left(\rho_{0}\right)$ for any $T \in G_{i}$, so $d\left(\pi \circ \psi_{\rho_{0}}\right)_{e}=0$. On the other hand, as we noted above, the image of the differential $\left(d \psi_{\rho_{0}}\right)_{e}$ is the space $B=B^{1}\left(\Gamma, \tilde{\mathfrak{g}}_{i}\right)$, where $\tilde{\mathfrak{g}}_{i}$ is the Lie algebra of $G_{i}$ with $\Gamma$-action given by Ad $\circ \rho_{0}$. Since $\rho_{0}$ is a simple point, it lies on a unique irreducible component of $R^{\prime}\left(\Gamma, G_{i}\right)$, so it follows that the image of $\psi_{\rho_{0}}$ (i.e., the orbit of $\rho_{0}$ ) is contained in $V$. Consequently, (11) factors through

$$
T_{\rho_{0}}(V) / B \rightarrow T_{\pi\left(\rho_{0}\right)}(W) .
$$

Since obviously $\operatorname{dim}_{K} T_{\rho_{0}}(V) \leq \operatorname{dim}_{K} T_{\rho_{0}}\left(\Re\left(\Gamma, G_{i}\right)\right)$ and

$$
T_{\rho_{0}}\left(\mathfrak{R}\left(\Gamma, G_{i}\right)\right) \simeq Z^{1}(\Gamma, \tilde{\mathfrak{g}})
$$

we therefore obtain that

$$
\operatorname{dim} X_{n}(\Gamma)=\operatorname{dim} W \leq \operatorname{dim}_{K} H^{1}\left(\Gamma, \tilde{\mathfrak{g}}_{i}\right) .
$$

Thus, the proof of Theorem 2 is now reduced to considering the following situation. Suppose $\rho_{0}: \Gamma \rightarrow \mathrm{GL}_{n}(K)$ is a completely reducible representation, set $\mathscr{G}=\overline{\rho_{0}(\Gamma)}$ (note that the connected component $\mathscr{G}^{\circ}$ is semisimple), and let $\tilde{\mathfrak{g}}$ be the Lie algebra of $\mathscr{G}$, considered as a $\Gamma$-module via $\mathrm{Ad} \circ \rho_{0}$. We need to give an upper bound on $\operatorname{dim}_{K} H^{1}(\Gamma, \tilde{\mathfrak{g}})$. This will be made more precise in Proposition 4.4 below after some preparatory remarks. 
First, notice that for the purpose of estimating $\operatorname{dim}_{K} H^{1}(\Gamma, \tilde{\mathfrak{g}})$, we may compose $\rho_{0}$ with the adjoint representation and assume without loss of generality that the group $\varphi$ is adjoint. Now, since $\varphi^{\circ}$ is semisimple, $\rho_{0}$ has a standard description by [Rapinchuk 2011, Theorem 6.7], i.e., there exist a commutative finite-dimensional $K$-algebra $A_{0}$, a ring homomorphism

$$
f_{0}: R \rightarrow A_{0}
$$

with Zariski-dense image, and a morphism of algebraic groups

$$
\theta: G\left(A_{0}\right) \rightarrow \mathscr{G}
$$

such that on a suitable finite-index subgroup $\Delta \subset \Gamma$, we have

$$
\left.\rho_{0}\right|_{\Delta}=\left.\left(\theta \circ F_{0}\right)\right|_{\Delta},
$$

where $F_{0}: \Gamma \rightarrow G\left(A_{0}\right)$ is the group homomorphism induced by $f_{0}$. Moreover, it follows from [Rapinchuk 2011, Proposition 5.3] that $\theta\left(G\left(A_{0}\right)\right)=\varphi^{\circ}$.

Next, let $\mathscr{G}_{1}, \ldots, \mathscr{G}_{r}$ be the (almost) simple components of $\mathscr{G}^{\circ}$ [Borel 1991, Proposition 14.10]. Since $\mathscr{G}^{\circ}$ is adjoint, the product map

$$
\varphi_{1} \times \cdots \times \mathscr{G}_{r} \rightarrow \mathscr{\varphi}^{\circ}
$$

is an isomorphism. The following lemma gives a more detailed description of $A_{0}$ :

Lemma 4.2. The algebraic ring $A_{0}$ is isomorphic to the product $\underbrace{K \times \cdots \times K}_{r \text { copies }}$.

Proof. Let $J_{0}$ be the Jacobson radical of $A_{0}$. Since $\mathscr{G}^{\circ}$ is semisimple (in particular, reductive), $J_{0}=\{0\}$ by [Rapinchuk 2011, Lemma 5.7], and consequently by [Rapinchuk 2011, Proposition 2.20], we have

$$
A_{0} \simeq K^{(1)} \times \cdots \times K^{(s)},
$$

where $K^{(i)} \simeq K$ for all $i$. Thus, $G\left(A_{0}\right)=G\left(K^{(1)}\right) \times \cdots \times G\left(K^{(s)}\right)$. As we observed above, the map $\theta$ is surjective, so since $G(K)$ is an almost simple group, it follows that $s \geq r$. On the other hand, by [Rapinchuk 2011, Theorem 3.1], for each root $\alpha \in \Phi$, there exists an injective map $\psi_{\alpha}: A_{0} \rightarrow \mathscr{G}$ such that

$$
\theta\left(e_{\alpha}(a)\right)=\psi_{\alpha}(a)
$$

where $e_{\alpha}\left(A_{0}\right)$ is the 1-parameter root subgroup of $G\left(A_{0}\right)$ corresponding to the root $\alpha$ [Rapinchuk 2011, Proposition 4.2]. Now if $s>r$, then $\theta$ would kill some simple component $G\left(K^{(i)}\right)$ of $G\left(A_{0}\right)$. Since $G\left(K^{(i)}\right)$ intersects each root subgroup $e_{\alpha}\left(A_{0}\right)$, the maps $\psi_{\alpha}$ would not be injective, a contradiction. So $s=r$, as claimed. 
Thus, we can write $f_{0}: R \rightarrow A_{0}$ as

$$
f_{0}(t)=\left(f_{0}^{(1)}(t), \ldots, f_{0}^{(r)}(t)\right)
$$

for some ring homomorphisms $f_{0}^{(i)}: R \rightarrow K$.

Remark 4.3. Notice that for each $i$, the image $\theta\left(G\left(K^{(i)}\right)\right)$ intersects a unique simple factor of $\varphi^{\circ}$, say $\theta\left(G\left(K^{(i)}\right)\right) \cap \varphi_{i} \neq\{e\}$, and then $\theta\left(G\left(K^{(i)}\right)\right)=\mathscr{G}_{i}$. Furthermore, it follows from the proof of Lemma 4.2 that $\theta$ is an isogeny, so since char $K=0$, the differential $(d \theta)_{e}: \mathfrak{g} \rightarrow \tilde{\mathfrak{g}}_{i}$ gives an isomorphism of Lie algebras. In particular, we see that the Lie algebras of all the simple factors $\varphi_{i}$ are isomorphic (in fact, they are isomorphic as $G(K)$-modules with $G(K)$ acting via $\operatorname{Ad} \circ \theta$ ).

To formulate the next result, we need to introduce some notation. Suppose $g: R \rightarrow K$ is a ring homomorphism. Then we will let $\operatorname{Der}^{g}(R, K)$ denote the space of $K$-valued derivations of $R$ with respect to $g$, i.e., an element $\delta \in \operatorname{Der}^{g}(R, K)$ is a map $\delta: R \rightarrow K$ such that for any $r_{1}, r_{2}, \in R$,

$$
\delta\left(r_{1}+r_{2}\right)=\delta\left(r_{1}\right)+\delta\left(r_{2}\right) \quad \text { and } \quad \delta\left(r_{1} r_{2}\right)=\delta\left(r_{1}\right) g\left(r_{2}\right)+g\left(r_{1}\right) \delta\left(r_{2}\right) .
$$

Proposition 4.4. Suppose $\rho_{0}: \Gamma \rightarrow \mathrm{GL}_{n}(K)$ is a linear representation, and set $\mathscr{G}=\overline{\rho_{0}(\Gamma)}$. Let $\tilde{\mathfrak{g}}$ denote the Lie algebra of $\mathscr{G}$, and assume $\mathscr{G}^{\circ}$ is semisimple. Then

$$
\operatorname{dim}_{K} H^{1}(\Gamma, \tilde{\mathfrak{g}}) \leq \sum_{i=1}^{r} \operatorname{dim}_{K} \operatorname{Der}_{0}^{f_{0}^{(i)}}(R, K),
$$

where the $f_{0}^{(i)}$ are the ring homomorphisms appearing in (17).

We first note two facts that will be needed in the proof. Let $\Lambda \subset \Gamma$ be any finiteindex subgroup. Then, as we have already seen, the space of 1 -cocycles $Z^{1}(\Lambda, \tilde{\mathfrak{g}})$ can be naturally identified with the tangent space

$$
T_{\rho_{0}}(\mathfrak{R}(\Lambda, \mathscr{G}))=\left\{\rho \in \operatorname{Hom}(\Lambda, \mathscr{G}(K[\varepsilon])) \mid \mu \circ \rho=\rho_{0}\right\} .
$$

Also observe that the restriction map

$$
\operatorname{res}_{\Gamma / \Lambda}: H^{1}(\Gamma, \tilde{\mathfrak{g}}) \rightarrow H^{1}(\Lambda, \tilde{\mathfrak{g}})
$$

is injective. Indeed, since $[\Gamma: \Lambda]<\infty$, the corestriction map

$$
\operatorname{cor}_{\Gamma / \Lambda}: H^{1}(\Lambda, \tilde{\mathfrak{g}}) \rightarrow H^{1}(\Gamma, \tilde{\mathfrak{g}})
$$

is defined and the composition $\operatorname{cor}_{\Gamma / \Lambda} \circ \operatorname{res}_{\Gamma / \Lambda}$ coincides with multiplication by $[\Gamma: \Lambda]$. Since char $K=0$, the injectivity of $\operatorname{res}_{\Gamma / \Lambda}$ follows.

Proof of Proposition 4.4. Set

$$
X=\operatorname{Der}_{0}^{(1)}(R, K) \oplus \cdots \oplus \operatorname{Der}^{f_{0}^{(r)}}(R, K),
$$


and let $\Delta \subset \Gamma$ be the finite-index subgroup appearing in (15). We will show that there exists a linear map $\psi: X \rightarrow H^{1}(\Delta, \tilde{\mathfrak{g}})$ such that

$$
\operatorname{res}\left(H^{1}(\Gamma, \tilde{\mathfrak{g}})\right) \subset \operatorname{im}(\psi) .
$$

The proposition then follows from the injectivity of the restriction map.

The map $\psi$ is constructed as follows. Choose derivations $\delta_{i} \in \operatorname{Der}_{0}^{f_{0}^{(i)}}(R, K)$ for $i=1, \ldots, r$, and let

$$
B=\underbrace{K[\varepsilon] \times \cdots \times K[\varepsilon]}_{r \text { copies }}
$$

(with $\varepsilon^{2}=0$ ). Then

$$
f_{\delta_{1}, \ldots, \delta_{r}}: R \rightarrow B, \quad s \mapsto\left(f_{0}^{(1)}(s)+\delta_{1}(s) \varepsilon, \ldots, f_{0}^{(r)}(s)+\delta_{r}(s) \varepsilon\right)
$$

is a ring homomorphism and hence induces a group homomorphism

$$
F_{\delta_{1}, \ldots, \delta_{r}}: \Gamma \rightarrow G(B)
$$

(recall that $\Gamma=E(R) \subset G(R)$ ). On the other hand, we have

$$
G(B) \simeq(\mathfrak{g} \oplus \cdots \oplus \mathfrak{g}) \rtimes(G(K) \times \cdots \times G(K)) \simeq \operatorname{Lie}\left(G\left(A_{0}\right)\right) \rtimes G\left(A_{0}\right)
$$

and

$$
\mathscr{G}(K[\varepsilon]) \simeq \tilde{\mathfrak{g}} \rtimes \mathscr{G},
$$

so we can define a group homomorphism $\tilde{\theta}: G(B) \rightarrow \mathscr{G}(K[\varepsilon])$ by the formula

$$
(x, g) \mapsto\left((d \theta)_{e}(x), \theta(g)\right),
$$

where $\theta: G\left(A_{0}\right) \rightarrow \mathscr{G}$ is the morphism appearing in (14). Notice that since by Remark 4.3, the differential of $\theta$ gives a homomorphism

$$
(d \theta)_{e}: \mathfrak{g} \rightarrow \tilde{\mathfrak{g}}_{i}
$$

for each factor $\mathfrak{g}$ of $\operatorname{Lie}\left(G\left(A_{0}\right)\right)$, the map $\tilde{\theta}$ can also be described as follows. Let $x_{1}, \ldots, x_{r} \in \mathfrak{g}$ and $g \in G\left(A_{0}\right)$. Then

$$
\tilde{\theta}\left(x_{1}, \ldots, x_{r}, g\right)=\left(\sum_{i=1}^{r}(d \theta)_{e}\left(x_{i}\right), \theta(g)\right) .
$$

Now, $\tilde{\theta} \circ F_{\delta_{1}, \ldots, \delta_{r}}$ is a homomorphism $\Gamma \rightarrow \mathcal{G}(K[\varepsilon])$, and in view of (15), we have

$$
\mu \circ\left(\left.\tilde{\theta} \circ F_{\delta_{1}, \ldots, \delta_{r}}\right|_{\Delta}\right)=\rho_{0} .
$$

It follows from (18) that

$$
c_{\delta_{1}, \ldots, \delta_{r}}:=\left.\tilde{\theta} \circ \operatorname{pr} \circ F_{\delta_{1}, \ldots, \delta_{r}}\right|_{\Delta},
$$


where pr: $G(B) \rightarrow \operatorname{Lie}\left(G\left(A_{0}\right)\right)$ is the projection, is an element of $Z^{1}(\Delta, \tilde{\mathfrak{g}})$. Now put

$$
\psi\left(\left(\delta_{1}, \ldots, \delta_{r}\right)\right)=\left[c_{\delta_{1}, \ldots, \delta_{r}}\right],
$$

where $\left[c_{\delta_{1}, \ldots, \delta_{r}}\right]$ denotes the class of $c_{\delta_{1}, \ldots, \delta_{r}}$ in $H^{1}(\Delta, \tilde{\mathfrak{g}})$.

Let us now turn to the proof of the inclusion (19). Suppose $\rho: \Gamma \rightarrow \mathscr{G}(K[\varepsilon])$ is a homomorphism with $\mu \circ \rho=\rho_{0}$. By [Rapinchuk 2011, Proposition 2.14, Theorem 3.1], we can associative to $\rho$ a commutative finite-dimensional $K$-algebra $A$ together with a ring homomorphism $f: R \rightarrow A$ with Zariski-dense image.

Lemma 4.5. Let $A$ be the finite-dimensional commutative $K$-algebra associated to $\rho$. Then

$$
A \simeq \tilde{K}^{(1)} \times \cdots \times \tilde{K}^{(r)},
$$

where, as above, $r$ is the number of simple components of $\varphi^{\circ}$ and, for each $i, \tilde{K}^{(i)}$ is isomorphic to either $K$ or $K[\varepsilon]\left(\right.$ with $\left.\varepsilon^{2}=0\right)$.

Proof. Let $J$ be the Jacobson radical of $A$. Since the unipotent radical $U$ of $\overline{\rho(\Gamma)}{ }^{\circ}$ is commutative (which follows from the fact that $\tilde{\mathfrak{g}}$ is the unipotent radical of $\mathscr{G}(K[\varepsilon]$ )), we have $J^{2}=\{0\}$ by [Rapinchuk 2011, Lemma 5.7]. Now by our assumption, $\mu \circ \rho=\rho_{0}$, where $\mu: \mathscr{G}(K[\varepsilon]) \rightarrow \mathscr{G}(K)$ is the homomorphism induced by ring homomorphism $K[\varepsilon] \rightarrow K, \varepsilon \mapsto 0$. In particular, for any root $\alpha \in \Phi$, we have

$$
\mu\left(\rho\left(e_{\alpha}(r)\right)\right)=\rho_{0}\left(e_{\alpha}(r)\right)
$$

for all $r \in R$. Since $\mu$ is a morphism of algebraic groups and the algebraic rings $A$ and $A_{0}$ associated to $\rho$ and $\rho_{0}$, respectively, are by construction the connected components of $\overline{\rho\left(e_{\alpha}(R)\right)}$ and $\overline{\rho_{0}\left(e_{\alpha}(R)\right)}$ for any root $\alpha$ [Rapinchuk 2011, Theorem 3.1], it follows that $\mu$ induces a surjective map $v: A \rightarrow A_{0}$. Moreover, since (20) holds for all roots $\alpha \in \Phi$, the construction of the ring operations on $A$ and $A_{0}$ given in [Rapinchuk 2011, Theorem 3.1] implies that $v$ is actually a ring homomorphism. Also notice that since $J$ is commutative and nilpotent, we have $J \subset \operatorname{ker} v$ by the definition of $\mu$. On the other hand, the ring $A_{0}$ is semisimple by Lemma 4.2, so $J=\operatorname{ker} v$. Thus, $A_{0} \simeq A / J \simeq K \times \cdots \times K$.

Next, by the Wedderburn-Malcev theorem, we can find a semisimple subalgebra $\tilde{B} \subset A$ such that $A=\tilde{B} \oplus J$ as $K$-vector spaces and $\tilde{B} \simeq A / J \simeq K \times \cdots \times K$ as $K$-algebras [Pierce 1982, Corollary 11.6]. Let $e_{i} \in \tilde{B}$ be the $i$-th standard basis vector. Since $e_{1}, \ldots, e_{r}$ are idempotent and we have $e_{1}+\cdots+e_{r}=1$ and $e_{i} e_{j}=0$ for $i \neq j$, it follows that we can write $A=\bigoplus_{i=1}^{r} A_{i}$, where $A_{i}=e_{i} A$. Clearly, $A_{i}=\tilde{B}_{i} \oplus J_{i}$ with $\tilde{B}_{i}=e_{i} \tilde{B} \simeq K$ and $J_{i}=e_{i} J$; in particular, $A_{i}$ is a local $K$-algebra with maximal ideal $J_{i}$ such that $J_{i}^{2}=\{0\}$. To complete the proof, it obviously suffices to show that $s_{i}:=\operatorname{dim}_{K} J_{i} \leq 1$ for all $i$. 
Now, by [Rapinchuk 2011, Proposition 6.5], for each $i=1, \ldots, r$, we have a Levi decomposition

$$
G\left(A_{i}\right)=\underbrace{(\mathfrak{g} \oplus \cdots \oplus \mathfrak{g})}_{s_{i} \text { copies }} \rtimes G(K),
$$

where $\mathfrak{g}$ is the Lie algebra of $G(K)$. Also, by [Rapinchuk 2011, Theorem 6.7], there exists a morphism

$$
\sigma: G(A) \rightarrow \mathscr{G}(K[\varepsilon])
$$

of algebraic groups such that for a suitable subgroup of finite index $\Delta^{\prime} \subset \Gamma$, we have

$$
\left.\rho\right|_{\Delta^{\prime}}=\left.\sigma \circ F\right|_{\Delta^{\prime}}
$$

where $F: \Gamma \rightarrow G(A)$ denotes the group homomorphism induced by $f$. Since $\mu \circ \rho=\rho_{0}$ and for $\tilde{\Delta}=\Delta \cap \Delta^{\prime}$, we have

$$
\left.\rho_{0}\right|_{\tilde{\Delta}}=\left.\left(\theta \circ F_{0}\right)\right|_{\tilde{\Delta}} \quad \text { and }\left.\quad \rho\right|_{\tilde{\Delta}}=\left.\sigma \circ F\right|_{\tilde{\Delta}}
$$

by (15) and (22), it follows that the diagram

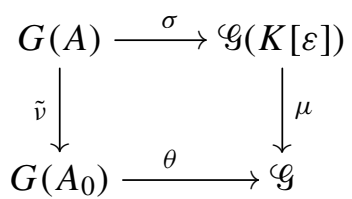

commutes (where $\tilde{v}$ is the homomorphism induced by $v$ ). Now Remark 4.3, together with the definition of $v$, implies that $(\theta \circ \tilde{v})\left(G\left(A_{i}\right)\right)=\varphi_{i}$, where $\varphi_{i}$ is a simple factor of $\mathscr{G}$. Since $G\left(A_{i}\right)$ coincides with its commutator subgroup [Stein 1971, Corollary 4.4], we obtain that $\sigma\left(G\left(A_{i}\right)\right)$ is a subgroup of $\mathscr{G}(K[\varepsilon])$ that maps to $\mathscr{G}_{i}$ under $\mu$ and coincides with its commutator, so the fact that the simple factors

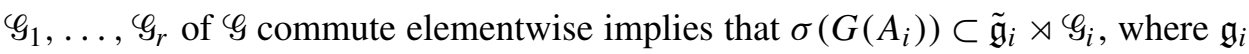
is the Lie algebra of $\varphi_{i}$. On the other hand, by [Rapinchuk 2011, Theorem 3.1], for each root $\alpha \in \Phi$, there exists an injective map $\tilde{\psi}_{\alpha}: A \rightarrow \mathscr{G}(K[\varepsilon])$ such that

$$
\sigma\left(e_{\alpha}(a)\right)=\tilde{\psi}_{\alpha}(a)
$$

where $e_{\alpha}(A)$ is the 1-parameter root subgroup of $G(A)$ corresponding to the root $\alpha$. So since $\tilde{\mathfrak{g}}_{i} \simeq \mathfrak{g}$ by Remark 4.3, the same argument as in the proof of Lemma 4.2 shows that $s_{i} \leq 1$.

For ease of notation, we will view $A$ as a subalgebra of

$$
\tilde{A}:=\underbrace{K[\varepsilon] \times \cdots \times K[\varepsilon] .}_{r \text { copies }}
$$


Then, using the lemma and the assumption that $\mu \circ \rho=\rho_{0}$, we can write the homomorphism $f: R \rightarrow A$ in the form

$$
f(t)=\left(f_{0}^{(1)}(t)+\delta_{1}(t) \varepsilon, \ldots, f_{0}^{(r)}(t)+\delta_{r}(t) \varepsilon\right)
$$

with $\left(\delta_{1}, \ldots, \delta_{r}\right) \in X$ and $\delta_{i}=0$ for $i=r_{2}+1, \ldots, r$.

To describe the cohomology class corresponding to $\rho$, we will now need to analyze more closely the morphism $\sigma$ introduced in (21). First, we note that if $\bar{A}=A / J$ and $G(A, J)$ is the congruence subgroup

$$
G(A, J)=\operatorname{ker}(G(A) \rightarrow G(\bar{A})),
$$

then by [Rapinchuk 2011, Proposition 6.5],

$$
G(A)=G(A, J) \rtimes G(\bar{A})
$$

is a Levi decomposition of $G(A)$. Now by [Borel 1991, Proposition 11.23], any two Levi subgroups of $(\mathscr{G}(K[\varepsilon]))^{\circ}$ are conjugate under an element of the unipotent radical $R_{u}(\mathscr{G}(K[\varepsilon]))^{\circ}$, which can be identified with $\varphi^{\circ}(K[\varepsilon],(\varepsilon)) \simeq \tilde{\mathfrak{g}}$. In our case, we can apply this to the groups $\sigma\left(G(\bar{A})\right.$ ) and $\theta\left(G\left(A_{0}\right)\right)=G^{\circ}$ (where $\theta$ is the morphism from (14)) to conclude that $B \theta\left(G\left(A_{0}\right)\right) B^{-1}=\sigma(G(\bar{A}))$ for some $B \in \mathscr{G}(K[\varepsilon],(\varepsilon)) \simeq \tilde{\mathfrak{g}}$. By direct computation, one sees that for any $X \in \mathscr{G}$ and $B=I+\varepsilon Y \in \mathscr{G}(K[\varepsilon],(\varepsilon))$,

$$
B X B^{-1}=\left(I+\varepsilon\left(Y-X Y X^{-1}\right)\right) X,
$$

which shows that

$$
\rho(\gamma)=\sigma(F(\gamma))=\left((\sigma \circ \operatorname{pr} \circ F)(\gamma)+Y-\operatorname{Ad}\left(\theta\left(F_{0}(\gamma)\right)(Y), \theta\left(F_{0}(\gamma)\right)\right)\right)
$$

for all $\gamma \in \tilde{\Delta}=\Delta \cap \Delta^{\prime}$ (where $\Delta$ and $\Delta^{\prime}$ are the finite-index subgroups of $\Gamma$ appearing in (15) and (22), respectively). Since $\theta\left(F_{0}(\gamma)\right)=\rho_{0}(\gamma)$ for $\gamma \in \tilde{\Delta}$, we can rewrite this as

$$
\rho(\gamma)=\left(c(\gamma), \rho_{0}(\gamma)\right)
$$

where

$$
c(\gamma)=(\sigma \circ \operatorname{pr} \circ F)(\gamma)+Y-\operatorname{Ad}\left(\rho_{0}(\gamma)\right)(Y) .
$$

Using (18), we obtain $c \in Z^{1}(\tilde{\Delta}, \tilde{\mathfrak{g}})$. Now let $b_{Y} \in B^{1}(\tilde{\Delta}, \tilde{\mathfrak{g}})$ be the 1-coboundary defined by $b_{Y}(\gamma)=Y-\operatorname{Ad}\left(\rho_{0}(\gamma)\right) Y$, and put $\tilde{c}=c-b_{Y}$ (thus, $\tilde{c}$ and $c$ define the same element of $\left.H^{1}(\tilde{\Delta}, \tilde{g})\right)$. Then

$$
\tilde{c}(\gamma)=(\sigma \circ \operatorname{pr} \circ F)(\gamma)
$$

for all $\gamma \in \tilde{\Delta}$. To complete the proof of the proposition, we will need the following: 
Lemma 4.6. Assume that $K$ is an algebraically closed field of characteristic 0 . Let $\pi: G \rightarrow \mathscr{G}^{\prime}$ be an isogeny of absolutely almost simple algebraic groups. Let $\mathfrak{g}$ and $\mathfrak{g}^{\prime}$ denote the Lie algebras of $\varphi$ and $\varphi^{\prime}$, respectively. Set

$$
\mathscr{H}=\mathfrak{g} \rtimes \mathscr{G} \text { and } \mathscr{H}^{\prime}=\mathfrak{g}^{\prime} \rtimes \mathscr{G}^{\prime},
$$

where $\varphi$ and $\mathscr{G}^{\prime}$ act on $\mathfrak{g}$ and $\mathfrak{g}^{\prime}$, respectively, via the adjoint representation. Then for any morphism $\varphi: \mathscr{H} \rightarrow \mathscr{H}^{\prime}$ such that $\varphi \mid \varphi=\pi$, there exists $a \in K$ such that

$$
\varphi(X, g)=\left(a(d \pi)_{e}(X), \pi(g)\right) .
$$

Proof. Since char $K=0$ and $\mathfrak{g}$ and $\mathfrak{g}^{\prime}$ are simple Lie algebras, the adjoint representations Ad: $\mathscr{G} \rightarrow \mathrm{GL}(\mathfrak{g})$ and $\mathrm{Ad}: \mathscr{G}^{\prime} \rightarrow \mathrm{GL}\left(\mathfrak{g}^{\prime}\right)$ are both irreducible. Let us now view $\mathfrak{g}^{\prime}$ as a $\mathscr{G}$-module with $\mathscr{G}$ acting via $\pi$. Then both $\left.\varphi\right|_{\mathfrak{g}}$ and $(d \pi)_{e}$ are $\mathscr{G}$-equivariant homomorphisms of irreducible $\mathscr{G}$-modules. So by Schur's lemma, $\left.\varphi\right|_{\mathfrak{g}}=a(d \pi)_{e}$ for some $a \in K$ [Artin 1991, Theorem 9.6].

Now, as above, we consider $A$ as a subalgebra of the algebra $\tilde{A}$ appearing in (25); after possible renumbering, we may assume that, in the notation of Lemma 4.5, we have $\tilde{K}^{(i)} \simeq K[\varepsilon]$ for $i=1, \ldots, s$, where $s=\operatorname{dim}_{K} J(A)$, and $\tilde{K}^{(i)} \simeq K$ for $i=s+1, \ldots, r$. We will view $G(A)$ as a subgroup of

$$
G(\tilde{A}) \simeq \operatorname{Lie}\left(G\left(A_{0}\right)\right) \rtimes G\left(A_{0}\right)
$$

and write $G\left(A_{0}\right)=G\left(K^{(1)}\right) \times \cdots \times G\left(K^{(r)}\right)$ and $\operatorname{Lie}\left(G\left(A_{0}\right)\right)=\mathfrak{g}_{1} \oplus \cdots \oplus g_{r}$, where $G\left(K^{(i)}\right)=G(K)$ and $\mathfrak{g}_{i}=\mathfrak{g}$ for all $i$. We will also regard $\sigma: G(A) \rightarrow G(K[\varepsilon])$ as a morphism $\sigma: G(\tilde{A}) \rightarrow \mathscr{G}(K[\varepsilon])$ with $\left.\sigma\right|_{\mathfrak{g}_{i}}=0$ for all $i>s$. Now since by our construction, the cocycles $c$ and $\tilde{c}$ lie in the same cohomology class, we may assume without loss of generality that $\sigma$ has the form

$$
\sigma\left(x_{1}, \ldots, x_{r}, g\right)=\left(\left.\sigma\right|_{\mathfrak{g}_{1} \oplus \cdots \oplus \mathfrak{g}_{r}}\left(x_{1}, \ldots, x_{r}\right), \theta(g)\right)
$$

for $\left(x_{1}, \ldots, x_{r}, g\right) \in\left(\mathfrak{g}_{1} \oplus \cdots \oplus \mathfrak{g}_{r}\right) \rtimes G(\bar{A})$. By Remark 4.3, for each factor $G\left(K^{(i)}\right)$ of $G\left(A_{0}\right)$, the differential $(d \theta)_{e}: \mathfrak{g}_{i} \rightarrow \tilde{\mathfrak{g}}_{i}$ yields an isomorphism of $G(K)$-modules (with $G(K)$ acting on $\tilde{\mathfrak{g}}_{i}$ via Ad $\circ \theta$ ). Furthermore, since $\left.\sigma\right|_{G(\bar{A})}=\theta$, the same argument as used in the proof of Lemma 4.5 shows that $\sigma\left(\mathfrak{g}_{i}\right)=\tilde{\mathfrak{g}}_{i}$ for $i=1, \ldots, s$. Now applying Lemma 4.6 to the restrictions $\left.\sigma\right|_{\mathfrak{g}_{i} \rtimes G\left(K^{(i)}\right)}$ and $\left.\left((d \theta)_{e}, \theta\right)\right|_{\mathfrak{g}_{i} \rtimes G\left(K^{(i)}\right)}$, we get

$$
\left.\sigma\right|_{\mathfrak{g}_{i}}=\left.a(d \theta)_{e}\right|_{\mathfrak{g}_{i}}
$$

for some $a \in K$ (possibly 0 ). Repeating for all factors shows that we have

$$
\left.\sigma\right|_{\mathfrak{g}_{1} \oplus \cdots \oplus \mathfrak{g}_{r}}\left(x_{1}, \ldots x_{r}\right)=\sum_{i=1}^{r} a_{i}(d \theta)_{e}\left(x_{i}\right)
$$


for $\left(x_{1}, \ldots, x_{r}\right) \in \mathfrak{g}_{1} \oplus \cdots \oplus \mathfrak{g}_{r}$. So replacing the element $\left(\delta_{1}, \ldots, \delta_{r}\right)$ in (26) by $\left(a_{1} \delta_{1}, \ldots, a_{r} \delta_{r}\right)$, we have

$$
\tilde{c}(\gamma)=c_{\delta_{1}, \ldots, \delta_{r}}(\gamma)
$$

for all $\gamma \in \tilde{\Delta}$. Now let $\psi\left(\left(\delta_{1}, \ldots, \delta_{r}\right)\right)=d_{\delta_{1}, \ldots, \delta_{r}} \in Z^{1}(\Delta, \tilde{\mathfrak{g}})$, and let $c_{\rho}$ be the element of $Z^{1}(\Gamma, \tilde{\mathfrak{g}})$ corresponding to $\rho$. It follows that

$$
\operatorname{res}_{\Delta / \tilde{\Delta}}\left(\operatorname{res}_{\Gamma / \Delta}\left(\left[c_{\rho}\right]\right)\right)=\operatorname{res}_{\Delta / \tilde{\Delta}}\left(\left[d_{\delta_{1}, \ldots, \delta_{r}}\right]\right),
$$

where

$$
\operatorname{res}_{\Gamma / \Delta}: H^{1}(\Gamma, \tilde{\mathfrak{g}}) \rightarrow H^{1}(\Delta, \tilde{\mathfrak{g}}) \quad \text { and } \quad \operatorname{res}_{\Delta / \tilde{\Delta}}: H^{1}(\Delta, \tilde{\mathfrak{g}}) \rightarrow H^{1}(\tilde{\Delta}, \tilde{\mathfrak{g}})
$$

are the restriction maps. So the injectivity of the restriction maps yields

$$
\operatorname{res}_{\Gamma / \Delta}\left(\left[c_{\rho}\right]\right)=\left[d_{\delta_{1}, \ldots, \delta_{r}}\right],
$$

which shows that

$$
\operatorname{res}\left(H^{1}(\Gamma, \tilde{\mathfrak{g}})\right) \subset \operatorname{im}(\psi) .
$$

This completes the proof of the proposition.

Proof of Theorem 2. In view of (12) and Proposition 4.4, it remains to show that $r \leq n$ and to give a bound on the dimension of the space $\operatorname{Der}^{g}(R, K)$, for any ring homomorphism $g: R \rightarrow K$, which is independent of $g$. Notice that $\mathscr{G}^{\circ} \subset \mathrm{GL}_{n}(K)$ and $\mathscr{G}^{\circ}=\mathscr{G}_{1} \times \cdots \times \mathscr{G}_{r}$, so we have

$$
n \geq \operatorname{rk} \mathscr{G}^{\circ}=\sum_{i=1}^{r} \operatorname{rk} \mathscr{G}_{i} \geq r
$$

as needed. For the second task, we have the following (elementary) lemma:

Lemma 4.7. Let $R$ be a finitely generated commutative ring, and let d denote the minimal number of generators of $R$ (i.e., the smallest integer such that there exists a surjection $\left.\mathbb{Z}\left[x_{1}, \ldots, x_{d}\right] \rightarrow R\right)$. Then for any field $K$ and ring homomorphism $g: R \rightarrow K, \operatorname{dim}_{K} \operatorname{Der}^{g}(R, K) \leq d$. If, moreover, $K$ is a field of characteristic $0, R$ is an integral domain with field of fractions $L$, and $g$ is injective, then $\operatorname{dim}_{K} \operatorname{Der}^{g}(R, K) \leq l$, where $l$ is the transcendence degree of $L$ over its prime subfield.

Proof. Let $S=\left\{r_{1}, \ldots, r_{d}\right\}$ be a minimal set of generators of $R$. Since any element $\delta \in \operatorname{Der}^{g}(R, K)$ is completely determined by its values on the elements of $S$, the map

$$
\delta \mapsto\left(\delta\left(r_{1}\right), \ldots, \delta\left(r_{d}\right)\right)
$$

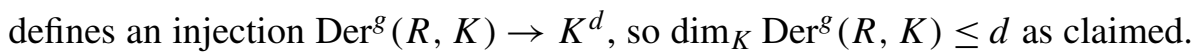

Now suppose that $R$ is a finitely generated integral domain and $g$ is injective. Since char $K=0$, after possibly localizing $R$ with respect to the multiplicative 
set $\mathbb{Z} \backslash\{0\}$ (which does not affect the dimension of the space $\operatorname{Der}^{g}(R, K)$ ), we can use Noether's normalization lemma to write $R$ as an integral extension of $S=\mathbb{Q}\left[x_{1}, \ldots, x_{l}\right]$ so that the field of fractions of $R$ is a separable extension of that of $S$. Combining this with the assumption that $g$ is injective, one easily sees that any derivation $\delta$ of $R$ is uniquely determined by its restriction to $S$ [Lang 2002, Chapter VII, Theorem 5.1], so in particular,

$$
\operatorname{dim}_{K} \operatorname{Der}^{g}(R, K) \leq \operatorname{dim}_{K} \operatorname{Der}^{g}(S, K)=: s .
$$

On the other hand, the argument given in the previous paragraph shows that $s \leq l$, which completes the proof.

Remark 4.8. Notice that the estimate $\operatorname{dim}_{K} \operatorname{Der}^{g}(R, K) \leq l$ may not be true if $g$ is not injective. Indeed, take $K=\overline{\mathbb{Q}}$, and let $R_{0}=\mathbb{Z}[X, Y]$ and $R=\mathbb{Z}[X, Y] /\left(X^{3}-Y^{2}\right)$. Furthermore, let

$$
f: \mathbb{Z}[X, Y] \rightarrow \overline{\mathbb{Q}}, \quad \varphi(X, Y) \mapsto \varphi(0,0)
$$

and $g: R \rightarrow \overline{\mathbb{Q}}$ denote the induced homomorphism. The space $\operatorname{Der}^{f}\left(R_{0}, \overline{\mathbb{Q}}\right)$ is spanned by the linearly independent derivations $\delta_{x}$ and $\delta_{y}$ defined by

$$
\delta_{x}(\varphi(X, Y))=\frac{\partial \varphi}{\partial X}(0,0) \quad \text { and } \quad \delta_{y}(\varphi(X, Y))=\frac{\partial \varphi}{\partial Y}(0,0),
$$

so $\operatorname{dim}_{\overline{\mathbb{Q}}} \operatorname{Der}^{f}\left(R_{0}, \overline{\mathbb{Q}}\right)=2$. Now notice that the natural map

$$
\operatorname{Der}^{g}(R, \overline{\mathbb{Q}}) \rightarrow \operatorname{Der}^{f}\left(R_{0}, \overline{\mathbb{Q}}\right)
$$

is bijective. Indeed, it is obviously injective, and since any $\delta \in \operatorname{Der}^{f}\left(R_{0}, \overline{\mathbb{Q}}\right)$ vanishes on the elements of the ideal $\left(X^{3}-Y^{2}\right) R_{0}$, it is also surjective. Thus, $\operatorname{dim}_{\overline{\mathbb{Q}}} \operatorname{Der}^{g}(R, \overline{\mathbb{Q}})=2$. On the other hand, if $L$ is the fraction field of $R$, then $l:=\operatorname{tr} \operatorname{deg}_{\mathbb{Q}} L$ is 1 .

\section{Applications to rigidity}

In this section, we will show how our results from [Rapinchuk 2011] imply various forms of classical rigidity for the elementary groups $E(\Phi, \mathcal{O})$, where $\Phi$ is a reduced irreducible root system of rank $>1$ and $\mathcal{O}$ is a ring of algebraic integers (or $S$ integers) in a number field. It is worth mentioning that all forms of rigidity ultimately boil down to the fact that $\mathcal{O}$ does not admit nontrivial derivations.

To fix notations, let $\Phi$ be a reduced irreducible root system of rank $>1, G$ the universal Chevalley-Demazure group scheme of type $\Phi$, and $\mathcal{O}$ a ring of algebraic $S$-integers in a number field $L$ such that $(\Phi, \mathcal{O})$ is a nice pair. Furthermore, let $\Gamma=E(\Phi, \mathcal{O})$ be the elementary subgroup of $G(\mathcal{O})$. 
Proposition 5.1. Let $\rho: \Gamma \rightarrow \mathrm{GL}_{m}(K)$ be an abstract linear representation over an algebraically closed field $K$ of characteristic 0 . Then there exist

(i) a finite-dimensional commutative $K$-algebra

$$
A \simeq K^{(1)} \times \cdots \times K^{(r)}
$$

with $K^{(i)} \simeq K$ for all $i$,

(ii) a ring homomorphism $f=\left(f^{(1)}, \ldots, f^{(r)}\right): \mathcal{O} \rightarrow A$ with Zariski-dense image, where each $f^{(i)}: \mathbb{O} \rightarrow K^{(i)}$ is the restriction to $\mathcal{O}$ of an embedding $\varphi_{i}: L \hookrightarrow K$ and $\varphi_{1}, \ldots, \varphi_{r}$ are all distinct, and

(iii) a morphism of algebraic groups $\sigma: G(A) \rightarrow \mathrm{GL}_{m}(K)$

such that for a suitable subgroup of finite index $\Delta \subset \Gamma$, we have

$$
\left.\rho\right|_{\Delta}=\left.\sigma\right|_{\Delta}
$$

Proof. Let $H=\overline{\rho(\Gamma)}$, where, as before, the bar denotes Zariski closure. We begin by showing that the connected component $H^{\circ}$ is automatically reductive. Suppose this is not the case, and let $U$ be the unipotent radical of $H^{\circ}$. Since the commutator subgroup $U^{\prime}=[U, U]$ is a closed normal subgroup of $H$, the quotient $\check{H}=H / U^{\prime}$ is affine, so we have a closed embedding $\iota: \check{H} \rightarrow \mathrm{GL}_{m^{\prime}}(K)$ for some $m^{\prime}$. Then $\check{\rho}=\iota \circ \pi \circ \rho$, where $\pi: H \rightarrow \check{H}$ is the quotient map, is a linear representation of

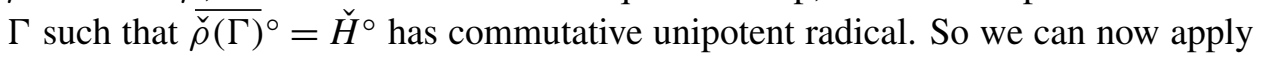
[Rapinchuk 2011, Theorem 6.7] to obtain a finite-dimensional commutative $K$ algebra $\check{A}$, a ring homomorphism $\check{f}: \mathcal{O} \rightarrow \check{A}$ (which is injective as any nonzero ideal in $O$ has finite index) with Zariski-dense image, and a morphism $\check{\sigma}: G(\check{A}) \rightarrow \check{H}$ of algebraic groups such that for a suitable finite-index subgroup $\check{\Delta} \subset \Gamma$, we have

$$
\left.\check{\rho}\right|_{\check{\Delta}}=\left.(\check{\sigma} \circ \check{F})\right|_{\check{\Delta}},
$$

where $\check{F}: \Gamma \rightarrow G(\check{A})$ is the group homomorphism induced by $\check{f}$.

Now let $J$ be the Jacobson radical of $\check{A}$. Since $\check{H}^{\circ}$ has commutative unipotent radical, $J^{2}=\{0\}$ by [Rapinchuk 2011, Lemma 5.7]. We claim that in fact $J=\{0\}$. Indeed, using the Wedderburn-Malcev theorem as in the proof of Lemma 4.5, we can write $\check{A}=\bigoplus_{i=1}^{r} \check{A}_{i}$, where for each $i, \check{A}_{i}=K \oplus J_{i}$ is a finite-dimensional local $K$-algebra with maximal ideal $J_{i}$ such that $J_{i}^{2}=\{0\}$. Then it suffices to show that $J_{i}=\{0\}$ for all $i$. So we may assume that $\check{A}$ is itself a local $K$-algebra of this form. Then, fixing a $K$-basis $\left\{\varepsilon_{1}, \ldots, \varepsilon_{s}\right\}$ of $J$, we have

$$
\check{f}(x)=f_{0}(x)+\delta_{1}(x) \varepsilon_{1}+\cdots+\delta_{s}(x) \varepsilon_{s},
$$

where $f_{0}: \mathcal{O} \rightarrow K$ is an injective ring homomorphism and $\delta_{1}, \ldots, \delta_{s} \in \operatorname{Der}^{f_{0}}(\mathbb{O}, K)$. On the other hand, since the fraction field of $\mathcal{O}$ is a number field, it follows from 
Lemma 4.7 that the derivations $\delta_{1}, \ldots, \delta_{s}$ are identically zero. So the fact that $\check{f}$ has Zariski-dense image forces $J=\{0\}$. Consequently, $\check{A} \simeq K \times \cdots \times K$.

Now by [Rapinchuk 2011, Proposition 5.3], $\check{\sigma}: G(\check{A}) \rightarrow \check{H}^{\circ}$ is surjective, so $\check{H}^{\circ}$ is semisimple and in particular reductive [Borel 1991, Proposition 14.10]. It follows that $U=[U, U]$ [Borel 1991, Corollary 14.11], and hence, being a nilpotent group, $U=\{e\}$, which contradicts our original assumption. Thus, $H^{\circ}$ must be reductive, as claimed.

We can now apply [Rapinchuk 2011, Theorem 6.7] to $\rho$ to obtain a finitedimensional commutative $K$-algebra $A$, a ring homomorphism $f: 0 \rightarrow A$ with Zariski-dense image, and a morphism $\sigma: G(A) \rightarrow H$ of algebraic groups such that for a suitable subgroup of finite index $\Delta \subset \Gamma$, we have

$$
\left.\rho\right|_{\Delta}=\left.(\sigma \circ F)\right|_{\Delta} .
$$

Moreover, the fact that $H^{\circ}$ is reductive implies that $A=K \times \cdots \times K$ [Rapinchuk 2011, Proposition 2.20, Lemma 5.7]. So we can write $f=\left(f^{(1)}, \ldots, f^{(r)}\right)$ for some ring homomorphisms $f^{(1)}, \ldots, f^{(r)}: \mathcal{O} \rightarrow K$. It is easy to see that all of the $f^{(i)}$ are injective, and since $L$ is the fraction field of $\mathcal{O}$, it follows that each homomorphism $f^{(i)}$ is a restriction to $\mathcal{O}$ of an embedding $\varphi_{i}: L \hookrightarrow K$. Finally, since $f$ has Zariski-dense image, all of the $\varphi_{i}$ must be distinct, completing the proof.

Keeping the notations of the proposition, we have the following:

Corollary 5.2. Any representation $\rho: \Gamma \rightarrow \mathrm{GL}_{m}(K)$ is completely reducible.

Proof. By Proposition 5.1, we have $\left.\rho\right|_{\Delta}=\left.\sigma\right|_{\Delta}$, so since $G(B)$ is a semisimple group and char $K=0,\left.\rho\right|_{\Delta}$ is completely reducible. Since $\Delta$ is a finite-index subgroup of $\Gamma$, it follows that $\rho$ is also completely reducible.

SS-rigidity and local rigidity. Notice that since by Lemma 4.7 there are no nonzero derivations $\delta: 0 \rightarrow K$, Proposition 4.4 and the estimate given in (12) yield that for $\Gamma=E(\Phi, \mathcal{O})$, we have $\operatorname{dim} X_{n}(\Gamma)=0$ for all $n \geq 1$, i.e., $\Gamma$ is $S S$-rigid. In fact, Corollary 5.2 implies that $\Gamma$ is locally rigid, that is, $H^{1}(\Gamma$, Ad $\rho)=0$ for any representation $\rho: \Gamma \rightarrow \mathrm{GL}_{m}(K)$. This is shown in [Lubotzky and Magid 1985], and we recall the argument for the reader's convenience. Let $V=K^{m}$. It is well known that

$$
H^{1}\left(\Gamma, \operatorname{End}_{K}(V, V)\right)=\operatorname{Ext}_{\Gamma}^{1}(V, V)
$$

[Lubotzky and Magid 1985, page 37], and $\operatorname{Ext}_{\Gamma}^{1}(V, V)=0$ by Corollary 5.2. But Ad $\circ \rho$, whose underlying vector space is $M_{m}(K)$, can be naturally identified as a $\Gamma$-module with $\operatorname{End}_{K}(V, V)$, so $H^{1}(\Gamma, \operatorname{Ad} \circ \rho)=0$, as claimed.

Superrigidity (compare [Bass et al. 1967, §16; Margulis 1991, Chapter VII]). Let $\Gamma=\mathrm{SL}_{n}(\mathbb{Z})(n \geq 3)$ and consider an abstract representation $\rho: \Gamma \rightarrow \mathrm{GL}_{m}(K)$. There exists a rational representation $\sigma: \mathrm{SL}_{n}(K) \rightarrow \mathrm{GL}_{m}(K)$ such that $\left.\rho\right|_{\Delta}=\left.\sigma\right|_{\Delta}$ for a 
suitable finite-index subgroup $\Delta \subset \Gamma$. Indeed, let $f: \mathbb{Z} \rightarrow A$ be the homomorphism associated to $\rho$. Since $A \simeq K^{(1)} \times \cdots \times K^{(r)}$ by Proposition 5.1, we see that $f$ is simply a diagonal embedding of $\mathbb{Z}$ into $K \times \cdots \times K$. But $f$ has Zariski-dense image, so $r=1$, and the rest follows.

Notice that for a general ring of $S$-integers $\mathcal{O}$, the algebraic group $G(A)$ that arises in Proposition 5.1 can be described as follows. Let $\mathscr{G}=R_{L / \mathbb{Q}}\left({ }_{L} G\right)$, where ${ }_{L} G$ is the algebraic group obtained from $G$ by extending scalars from $\mathbb{Q}$ to $L$ and $R_{L / \mathbb{Q}}$ is the functor of restriction of scalars. Then $\mathscr{G}(K) \simeq G(K) \times \cdots \times G(K)$ with the factors corresponding to all of the distinct embeddings of $L$ into $K$ [Platonov and Rapinchuk 1994, §2.1.2]. The group $G(A)$ is then obtained from $\mathscr{G}(K)$ by simply projecting to the factors corresponding to the embeddings $\varphi_{1}, \ldots, \varphi_{r}$, so any representation of $E(\Phi, \mathcal{O})$ factors through $\mathscr{G}$.

Remark 5.3. Let us point out that another situation in which $\operatorname{Der}^{f}(R, K)=0$ occurs is if $K$ is a field of characteristic $p>0$ and $R$ is a commutative ring of characteristic $p$ such that $R^{p}=R$. This allows one to use arguments similar to the ones presented in this section to recover results of Seitz [1997]. Details will be published elsewhere.

\section{Acknowledgments}

I would like to thank my advisor Professor G. A. Margulis for suggesting the problems considered in this paper and for a number of helpful discussions. I am indebted to G. Prasad for insightful conversations about pseudoreductive groups during my visit to the University of Michigan. I would also like to thank B. Conrad for detailed remarks on the connections between pseudoreductive groups and Conjecture (BT). Finally, I thank M. Kassabov for useful communications regarding algebraic rings.

I was supported by an NSF Postdoctoral Fellowship in the final stages of the preparation of this paper.

\section{References}

[Artin 1991] M. Artin, Algebra, Prentice Hall, Englewood Cliffs, NJ, 1991. MR 92g:00001 MR 0788:00001

[Bak and Rehmann 1982] A. Bak and U. Rehmann, "The congruence subgroup and metaplectic problems for $\mathrm{SL}_{n \geq 2}$ of division algebras", J. Algebra 78:2 (1982), 475-547. MR 85j:11041 Zbl 0495.20022

[Bass et al. 1967] H. Bass, J. Milnor, and J.-P. Serre, "Solution of the congruence subgroup problem for $\mathrm{SL}_{n}(n \geq 3)$ and $\mathrm{Sp}_{2 n}(n \geq 2)$ ", Inst. Hautes Études Sci. Publ. Math. 33 (1967), 59-137. MR 39 \#5574 Zbl 0174.05203

[Borel 1991] A. Borel, Linear algebraic groups, 2nd ed., Graduate Texts in Mathematics 126, Springer, New York, 1991. MR 92d:20001 Zbl 0726.20030

[Borel and Tits 1973] A. Borel and J. Tits, "Homomorphismes "abstraits" de groupes algébriques simples”, Ann. of Math. (2) 97 (1973), 499-571. MR 47 \#5134 Zbl 0272.14013 
[Conrad et al. 2010] B. Conrad, O. Gabber, and G. Prasad, Pseudo-reductive groups, New Mathematical Monographs 17, Cambridge University Press, 2010. MR 2011k:20093 Zbl 1216.20038

[Ershov et al. 2011] M. Ershov, A. Jaikin-Zapirain, and M. Kassabov, "Property (T) for groups graded by root systems", preprint, 2011. arXiv 1102.0031

[Farb and Dennis 1993] B. Farb and R. K. Dennis, Noncommutative algebra, Graduate Texts in Mathematics 144, Springer, New York, 1993. MR 94j:16001 Zbl 0797.16001

[Gille and Szamuely 2006] P. Gille and T. Szamuely, Central simple algebras and Galois cohomology, Cambridge Studies in Advanced Mathematics 101, Cambridge University Press, 2006. MR 2007k:16033 Zbl 1137.12001

[Greenberg 1964] M. J. Greenberg, "Algebraic rings", Trans. Amer. Math. Soc. 111 (1964), 472-481. MR 28 \#3040 Zbl 0135.21503

[Hahn and O'Meara 1989] A. J. Hahn and O. T. O'Meara, The classical groups and K-theory, Grundlehren Math. Wiss. 291, Springer, Berlin, 1989. MR 90i:20002 Zbl 0683.20033

[de la Harpe and Valette 1989] P. de la Harpe and A. Valette, La propriété (T) de Kazhdan pour les groupes localement compacts, Astérisque 175, Société Mathématique de France, Paris, 1989. MR 90m:22001 Zbl 0759.22001

[Humphreys 1975] J. E. Humphreys, Linear algebraic groups, Graduate Texts in Mathematics 21, Springer, New York, 1975. MR 53 \#633 Zbl 0325.20039

[van der Kallen 1976] W. van der Kallen, "Injective stability for $K_{2}$ ”, pp. 77-154 in Algebraic $K$-theory (Evanston, IL, 1976), edited by M. R. Stein, Lecture Notes in Mathematics 551, Springer, Berlin, 1976. MR 58 \#22243 Zbl 0349.18009

[Kassabov and Sapir 2009] M. Kassabov and M. V. Sapir, "Nonlinearity of matrix groups", J. Topol. Anal. 1:3 (2009), 251-260. MR 2010m:20078 Zbl 1189.20042

[Knus et al. 1998] M.-A. Knus, A. Merkurjev, M. Rost, and J.-P. Tignol, The book of involutions, Amer. Math. Soc. Colloq. Publ. 44, American Mathematical Society, Providence, RI, 1998. MR 2000a:16031 Zbl 0955.16001

[Lam 2001] T. Y. Lam, A first course in noncommutative rings, 2nd ed., Graduate Texts in Mathematics 131, Springer, New York, 2001. MR 2002c:16001 Zbl 0980.16001

[Lang 2002] S. Lang, Algebra, 3rd ed., Graduate Texts in Mathematics 211, Springer, New York, 2002. MR 2003e:00003 Zbl 0984.00001

[Lubotzky and Magid 1985] A. Lubotzky and A. R. Magid, Varieties of representations of finitely generated groups, Mem. Amer. Math. Soc. 58, 1985. MR 87c:20021 Zbl 0598.14042

[Margulis 1991] G. A. Margulis, Discrete subgroups of semisimple Lie groups, Ergeb. Math. Grenzgeb.

(3) 17, Springer, Berlin, 1991. MR 92h:22021 Zbl 0732.22008

[Matsumoto 1966] H. Matsumoto, "Subgroups of finite index in certain arithmetic groups", pp. 99103 in Algebraic groups and discontinuous subgroups (Boulder, CO, 1965), American Mathematical Society, Providence, RI, 1966. MR 34 \#4373 Zbl 0178.35302

[Milnor 1971] J. Milnor, Introduction to algebraic K-theory, Annals of Mathematics 72, Princeton University Press, 1971. MR 50 \#2304 Zbl 0237.18005

[Pierce 1982] R. S. Pierce, Associative algebras, Graduate Texts in Mathematics 88, Springer, New York, 1982. MR 84c:16001 Zbl 0497.16001

[Platonov and Rapinchuk 1994] V. Platonov and A. Rapinchuk, Algebraic groups and number theory, Pure and Applied Mathematics 139, Academic Press, Boston, MA, 1994. MR 95b:11039 Zbl 0841.20046 
[Rapinchuk 1998] A. S. Rapinchuk, "On SS-rigid groups and A. Weil's criterion for local rigidity, I", Manuscripta Math. 97:4 (1998), 529-543. MR 99m:20019 Zbl 0920.20004

[Rapinchuk 1999] A. Rapinchuk, "On the finite-dimensional unitary representations of Kazhdan groups”, Proc. Amer. Math. Soc. 127:5 (1999), 1557-1562. MR 99h:22004 Zbl 0926.22001

[Rapinchuk 2011] I. A. Rapinchuk, "On linear representations of Chevalley groups over commutative rings”, Proc. Lond. Math. Soc. (3) 102:5 (2011), 951-983. MR 2012e:20108 Zbl 1232.20049

[Rapinchuk 2013] I. A. Rapinchuk, "On the character varieties of finitely generated groups", preprint, 2013. arXiv 1308.2692

[Rapinchuk et al. 1996] A. S. Rapinchuk, V. V. Benyash-Krivetz, and V. I. Chernousov, "Representation varieties of the fundamental groups of compact orientable surfaces", Israel J. Math. 93 (1996), 29-71. MR 98a:57002 Zbl 0857.14012

[Seitz 1997] G. M. Seitz, "Abstract homomorphisms of algebraic groups", J. London Math. Soc. (2) 56:1 (1997), 104-124. MR 99b:20077 Zbl 0904.20038

[Stein 1971] M. R. Stein, "Generators, relations and coverings of Chevalley groups over commutative rings”, Amer. J. Math. 93 (1971), 965-1004. MR 48 \#437 Zbl 0246.20034

[Stein 1973] M. R. Stein, "Surjective stability in dimension 0 for $K_{2}$ and related functors", Trans. Amer. Math. Soc. 178 (1973), 165-191. MR 48 \#6267 Zbl 0267.18015

[Steinberg 1968] R. Steinberg, Lectures on Chevalley groups, Yale University, New Haven, CT, 1968. MR 57 \#6215 Zbl 1196.22001

Communicated by Brian Conrad

Received 2012-06-09 Revised 2012-06-15 Accepted 2012-09-07

rapinch@math.harvard.edu Department of Mathematics, Harvard University,

1 Oxford Street, Cambridge, MA, 02138, United States 


\section{Algebra \& Number Theory}

msp.org/ant

\section{EDITORS}

MANAGING EDITOR

Bjorn Poonen

Massachusetts Institute of Technology

Cambridge, USA

\author{
EDITORIAL BOARD CHAIR \\ David Eisenbud \\ University of California \\ Berkeley, USA
}

\section{BOARD OF EDITORS}

Georgia Benkart

Dave Benson

Richard E. Borcherds

John H. Coates

J-L. Colliot-Thélène

Brian D. Conrad

Hélène Esnault

Hubert Flenner

Edward Frenkel

Andrew Granville

Joseph Gubeladze

Roger Heath-Brown

Ehud Hrushovski

Craig Huneke

Mikhail Kapranov

Yujiro Kawamata

János Kollár

Yuri Manin

Barry Mazur

Philippe Michel
University of Wisconsin, Madison, USA

University of Aberdeen, Scotland

University of California, Berkeley, USA

University of Cambridge, UK

CNRS, Université Paris-Sud, France

University of Michigan, USA

Freie Universität Berlin, Germany

Ruhr-Universität, Germany

University of California, Berkeley, USA

Université de Montréal, Canada

San Francisco State University, USA

Oxford University, UK

Hebrew University, Israel

University of Virginia, USA

Yale University, USA

University of Tokyo, Japan

Princeton University, USA

Northwestern University, USA

Harvard University, USA

École Polytechnique Fédérale de Lausanne
Susan Montgomery

Shigefumi Mori

Raman Parimala

Jonathan Pila

Victor Reiner

Karl Rubin

Peter Sarnak

Joseph H. Silverman

Michael Singer

Vasudevan Srinivas

J. Toby Stafford

Bernd Sturmfels

Richard Taylor

Ravi Vakil

Michel van den Bergh

Marie-France Vignéras

Kei-Ichi Watanabe

Efim Zelmanov

Shou-Wu Zhang
University of Southern California, USA

RIMS, Kyoto University, Japan

Emory University, USA

University of Oxford, UK

University of Minnesota, USA

University of California, Irvine, USA

Princeton University, USA

Brown University, USA

North Carolina State University, USA

Tata Inst. of Fund. Research, India

University of Michigan, USA

University of California, Berkeley, USA

Harvard University, USA

Stanford University, USA

Hasselt University, Belgium

Université Paris VII, France

Nihon University, Japan

University of California, San Diego, USA

Princeton University, USA

PRODUCTION

production@msp.org

Silvio Levy, Scientific Editor

See inside back cover or msp.org/ant for submission instructions.

The subscription price for 2013 is US $\$ 200 /$ year for the electronic version, and $\$ 350 /$ year $(+\$ 40$, if shipping outside the US) for print and electronic. Subscriptions, requests for back issues and changes of subscribers address should be sent to MSP.

Algebra \& Number Theory (ISSN 1944-7833 electronic, 1937-0652 printed) at Mathematical Sciences Publishers, 798 Evans Hall \#3840, c/o University of California, Berkeley, CA 94720-3840 is published continuously online. Periodical rate postage paid at Berkeley, CA 94704, and additional mailing offices.

ANT peer review and production are managed by EditFLOW ${ }^{\circledR}$ from Mathematical Sciences Publishers.

\section{PUBLISHED BY}

- mathematical sciences publishers

nonprofit scientific publishing

http://msp.org/

(C) 2013 Mathematical Sciences Publishers 


\section{Algebra \& Number Theory}

$\begin{array}{lll}\text { Volume } 7 & \text { No. } 7 \quad 2013\end{array}$

Weil representation and transfer factor

TERUJI THOMAS

Analytic families of finite-slope Selmer groups

1571

JONATHAN POTTHARST

Multiplicative excellent families of elliptic surfaces of type $E_{7}$ or $E_{8}$

ABHINAV KUMAR and TETSUJI SHIODA

Cohomological invariants of algebraic tori

SAM Blinstein and AleXANDer MerkurJeV

On abstract representations of the groups of rational points of algebraic groups and their 1685 deformations

IGOR A. RAPINCHUK

Betti diagrams from graphs

AlEXANDER ENGSTRÖM and MATTHEW T. STAMPS

Hopf monoids from class functions on unitriangular matrices

Marcelo Aguiar, NANTEl Bergeron and Nathaniel Thiem 